\title{
A Spectrum Defragmentation Algorithm Using Jellyfish Optimization Technique in Elastic Optical Network (EON)
}

Selvakumar S ( $\sim$ selvakumar.s@vit.ac.in )

Vellore Institute of Technology: VIT University https://orcid.org/0000-0002-0269-198X

S S Manivannan

Vellore Institute of Technology: VIT University

\section{Research Article}

Keywords: EON, Spectrum Defragmentation, Blocking Probability, Proactive-Reactive approach, Jellyfish Search Optimization.

Posted Date: February 18th, 2021

DOI: https://doi.org/10.21203/rs.3.rs-207934/v1

License: (c) (i) This work is licensed under a Creative Commons Attribution 4.0 International License.

Read Full License 


\title{
A Spectrum Defragmentation Algorithm Using Jellyfish Optimization Technique in Elastic Optical Network (EON)
}

\author{
Selvakumar $S$ and Manivannan $S$ * \\ School of Information Technology and Engineering (SITE), \\ Vellore Institute of Technology, Vellore, India. \\ selvakumar.s@vit.ac.in and manivannan.ss@vit.ac.in* \\ (* Corresponding Author)
}

\begin{abstract}
The rapid growth of the technologies, high bandwidth applications and cloud data centers consume heavy internet service. So, the consumer of the internet expects a high capacity medium for communication. The Elastic Optical Network (EON) provides a flexible and reliable transmission service for the consumers. The spectrum fragmentation is a key challenge in EON. In simple terms, unaligned Frequency Slots (FSs) in the network are referred to as fragmented spectrum, while in defragmentation, the available FSs need to be rearranged to create room for the new connection requests. The problem in defragmentation occurs due to the lack of a continuous spectrum and it leads to depreciation in spectrum usage and simultaneously increasing the Blocking Probability (BP) which disrupts the majority of the existing connections in the network. Several techniques and approaches were suggested to reduce the possibility of disruption and reconfiguration in the network while defragmenting the frequency slots. This paper proposes a new algorithm to overcome the drawbacks and improvement in the quality of service of the network. The proposed algorithm holds the approach of proactive and reactive along with the meta-heuristic nature-inspired optimization technique called Jellyfish Search Optimization (JSO). The proposed combination, PR-DF-JFSO outperforms well in terms of spectrum utilization, network efficiency, and quality of service offered when compared to the state-of-the-art spectrum defragmentation algorithms according to the results of experiments done using standard quality metrics.
\end{abstract}

Keywords: EON, Spectrum Defragmentation, Blocking Probability, Proactive-Reactive approach, Jellyfish Search Optimization.

\section{INTRODUCTION}

In this technologically advanced era, the internet has become inevitable almost in all sectors. The internet needs a medium to transmit data from one place to another. As the internet got globally connected along all the edges of the world, it needs an efficient medium in terms of faster transmission, minimal data loss, and economics. The transmission of data over the network is done in the form of a light beam that passes the signal between the sender and receiver node in a telecommunication network called an optical network and it is otherwise known as a Flex grid optical network.

EON, flexible grid technology is introduced to overcome the challenges faced in fixed grid method. Due to the global increase of internet users across the globe, the existing Dense Wavelength Division Multiplexing (DWDM) technologies cannot cope with the massive demand for bandwidth under a fixed grid. EON was introduced to fix and overcome this issue by managing the bandwidth, increasing the channel capacity, and efficiently utilizing the optical spectrum. In EON, the available spectrum is divided into small spectral pieces called Frequency Slots (FSs). Each FSs have a certain wavelength, based on the bandwidth requirements, the number of spectral slices are allotted to a specific connection and release the unassigned slots for new connection requests. Figure 1 shows spectrum saving efficiency between the fixed, flex and gridless optical network. In fixed grid small amount of demand also occupied single channel space of $50 \mathrm{GHz}$ unnecessarily but flex grid channel spaces are allocated based on the demand with guard band. In gridless there is no specific guard band allocated it may or not allocated according to the demand. The flex and gridless network provides much better spectrum management and finer granularity of spectrum slots compared with the International Telecommunication Union Telecommunication (ITU-T) fixed grid network. When a new connection request arrives, the network needs to consider the routing path where the connection needs to be established and the available frequency slots in the network spectrum. Routing and Spectrum Allocation (RSA) plays a major role in EON for allotting and establishing a new connection to the network. Due to heavy traffic loads, RSA had become a quite challenging task to assign the frequency slots and routes without compromising the network efficiency and spectral utilization. Three RSA constraints need to be imposed in EON. 


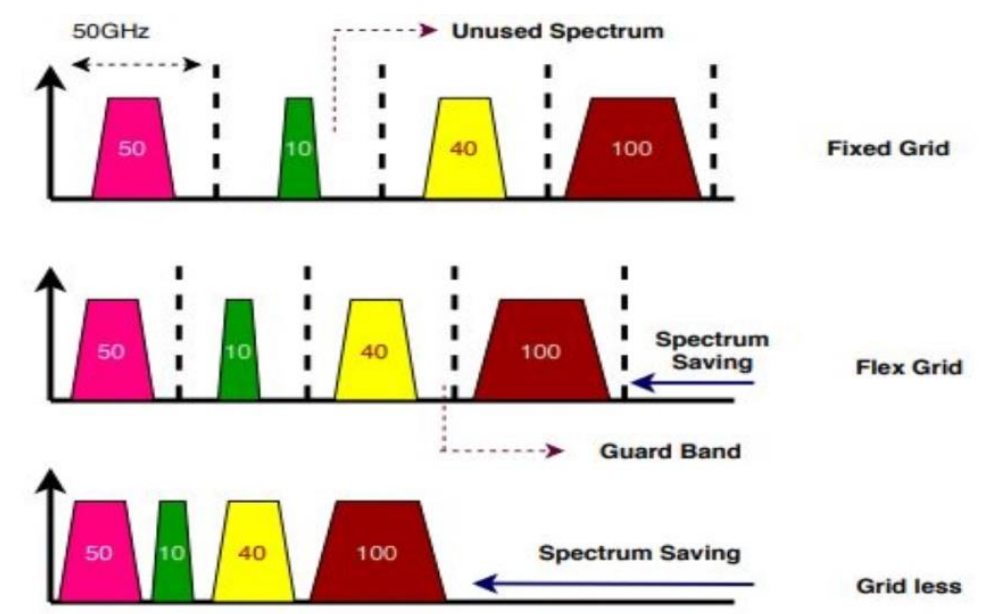

Figure 1.Difference between fixed, flex, and gridless network.

The RSA constraints are contiguous FSs, Spectrum Contiguity, and Spectral Conflict or nonoverlapping. Dynamic traffic load leads to frequent setting and closing up of connection often ends up in isolated FSs. These non-contiguous FSs in the network spectrum have a poor chance of getting allocated to a new connection. If more FSs went isolated in a network, and then there are more chances for the new connection request to get rejected thus leads to poor spectrum utilization shows in figure 2 . To overcome the fragmentation of the spectrum, Spectrum Defragmentation (DF) algorithms were employed to minimize the count of rejected connections in a network. DF is the process of reconfiguring the FSs in the spectral domain to make the FSs contiguous and helps in accepting new connection requests thus utilizes the full potential of the spectrum. In DF, there occurs a problem of disrupting the existing connection by nearly reconfiguring most of the allotted FSs thus suppresses the quality of service $(\mathrm{QoS})$ delivered by the overall network. It is suggestible to limit, minimize, and control the percent of reconfiguration of FSs in a spectrum for efficient utilization of the overall network without losing the existing connections for a new request.

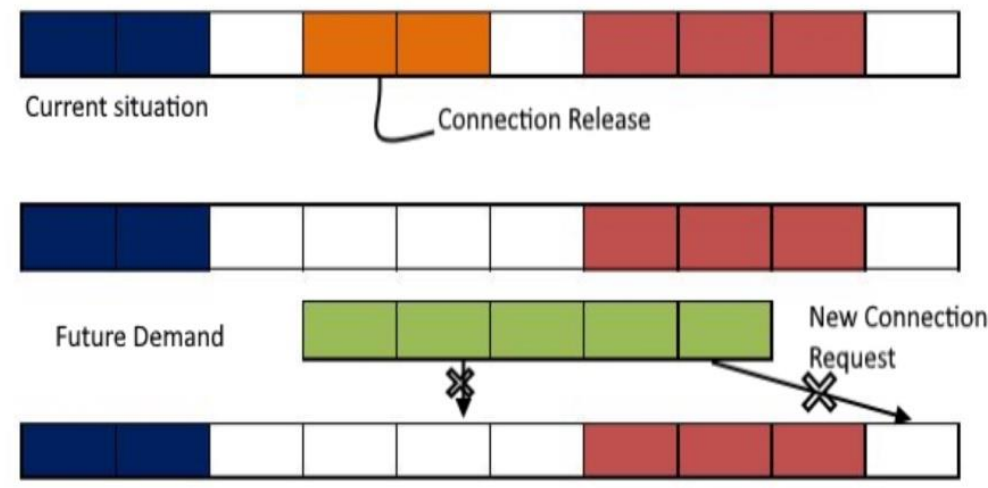

Figure 2. The Spectrum Fragmentation Problem in EON.

To fix and resolve the aforementioned issues in Fragmentation and DF in the spectrum, a hybrid optimization technique is introduced and that is to say proactive approach, reactive approach, and Jellyfish Search Optimization was combined to call it as PR-DF-JFSO. Under proactive and reactive approaches, various DF algorithms exist based upon the network requirement and necessity. Jellyfish Search Optimization is a metaheuristic, nature-inspired algorithm based on the food searching habits and migration movements of the jellyfish in the ocean currents. The performance of the proposed work is evaluated using some standard quality metrics called Blocking Probability, Bandwidth Frequency Ratio, and Spectrum Utilization Gain and the results will then be compared with the state-of-the-art DF algorithms to prove the significance of the work done.

The rest of the work is structured as follows. Section 2 discusses the existing works related to this study. A brief theoretical explanation about the proposed technique is presented in Section 3. Section 4 comprises the evaluation of the proposed work with the help of performance metrics, experimental results, and comparison illustrations. The final summary and future scope discussion of the work will be taken place in Section 5 . 


\section{BACKGROUND STUDY}

In this section, the prevailing literature is examined by performing an analysis on it. The related papers mainly evaluate the various approaches and algorithms to improve the performance of spectrum defragmentation and spectrum allocation. The routing and allocation of spectrum is a major challenge in EON. Non-contiguous spectrum allocation and fragment the spectrum into small portion leads to the result of inflation in blocking probability and inefficiency in spectrum utilization. To overwhelm the drawbacks, this paper utilizes the existing K- Shortest Path (The Floyd Warshall and Bellman-Ford) [1-3] for routing and proposed a new defragmentation algorithm to reduce fragmentation complexity. In [4] authors were presented two algorithms that reduce fragmentation issues on assigning the spectrum over a new connection request. The Least Load Routing and Spectrum Assignment (LLRSA) selecting a communication path by using k alternate routes in the network. So it can avoid the unnecessary blocking of the new connection request however when the new connection request arrives the existing available FSs index is calculated to assign each request. The next algorithm is route fragmentation aware RSA which is assigned to the connection only when the maximum available contiguous slot present in that route.

Fragmentation aware spectrum allocation algorithm is employed by partitioning the spectrum into small blocks or segments based on the request. When a request requires $\mathrm{n}$ number of FSs, and the acquirable block has $m$ Frequency Slots, then it has $m-n+1$ sub-segments of the spectrum. The initialization of all variables should be initialized at the beginning of the algorithm. B denotes the number of blocks, $\mathrm{K}_{\mathrm{i}}$ represents the shortest path and $\mathrm{CB}$ denotes the minimum cost of the block. The $\mathrm{B}$ and $\mathrm{CB}$ are initialized by 0 and $\infty$. When the system receives the connection request, it checks with the number of blocks and shortest path, if the $\mathrm{H}<\mathrm{K}_{\mathrm{i}}$, then it checks with the cost of the blocks, if the cost of the blocks is less than $\infty$, then the connection request is accepted otherwise it is blocked. This algorithm is executed in US network and NSF network and assesses the performance of bandwidth blocking probability and spectrum utilization and the total number of hops [5].

Defragmentation based Load Balancing Routing and Spectrum Assignment (DLBRSA) algorithm performs the reconfiguration on the previous connection and selecting the suitable path from the shortest path and selects the path which has the mass of a continuous slot of frequency during spectrum defragmentation and decreases the usage of network resource. After the termination of each connection the deallocation of the resource is required, if the spectrum in the fragment remains unused and it results to demand in bandwidth. It reassigns the network traffic and is loaded with the minimum path. This technique acquires better performance on the utilization of network resources and performs the load balancing of the spectrum allocation and assures the path distance of the connection request is the minimum [6].

Proactive Defragmentation (PD) algorithm is executed every $\mathrm{N}$ time units and finds out the values of $\mathrm{N}$. $\mathrm{N}$ refers to the period of defragmentation, the result of defragmentation is based on the aspects of the load and the size of the network. When network paths are determined, considering the shortest path is used frequently than other paths. Higher slot first index first is used to reallocate the connections preferably more on the right side of the spectrum. When the connections are moved out to left, the usage of the spectrum in the network connection is condensed [7].

The genetic and ant colony based defragmentation algorithms are reduced the number of connection blocking under the dynamic traffic conditions with unicast communication. The proactive defragmentation algorithm was designed and executed with two new metrics to avoid fragmentation and future blocks in the network. The DF-Gen and D-Ant reduce the bandwidth blocks and increases the spectrum utilization [8]. In [9] both the pro and the reactive algorithm presented and results are compared with sequential blocking, fragmentation ratio, and entropy of each request treat fewer than three dynamic situations. The ProactiveReactive Defragmentation (PRDEF) algorithm works (PRDEF) very effectively compared with other heuristicbased approaches Fragmentation Aware RSA(FA-RSA) [10], Holding Time-Aware Routing algorithm(HTAR) [11], and Hitless Optical Path Shift (HOPS) [12].

This work evaluates and correlates the various spectrum defragmentation techniques. The following are some of spectrum defragmentation techniques: Make-before-Break, Hitless Defragmentation, Spectrum Sweeping Retuning, Spectrum Conversion, Multi-path Routing, and Fa-RSA. The above-mentioned techniques are compared related to the fragmentation time, interruption of service, and requires additional devices. All the techniques have some merits and demerits [13]. However, the Fa-RSA method doesn't require any additional devices, owing to the fact it is generic and feasible than other techniques. 

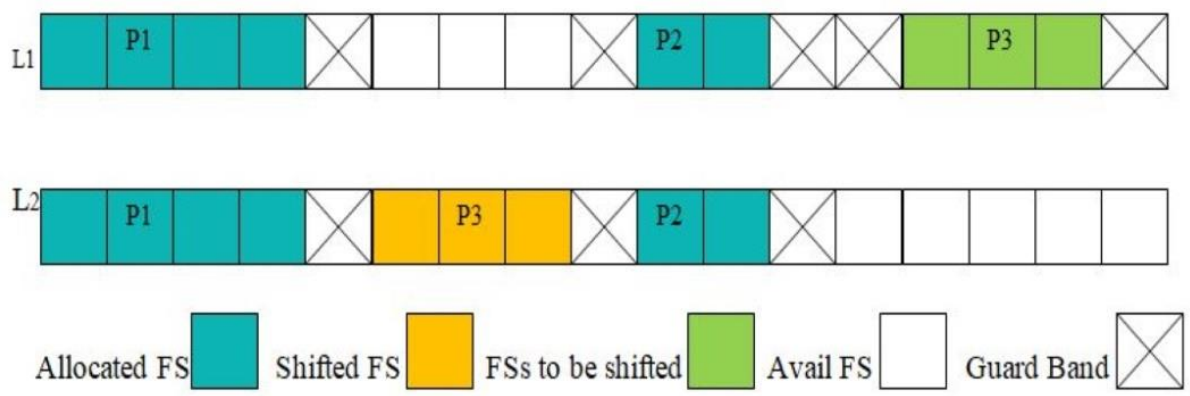

Figure 3. The Spectrum Defragmentation in EON

In the proactive defragmentation approach, the unused spectrum in the network is defragmented either periodically or proactively by some certain event on the network. The Bandwidth Blocking Probability is reduced while defragmenting the spectrum periodically by reducing the reallocation of the previous connections throughout the entire path of the network. The traffic load of the network is balanced concurrently. The numerous proposals have explored the way to reduce the reallocation of connections. In the reactive defragmentation approach, the reallocation of connections is done only when it is necessary because it accepts the connection when the network path has an appropriate room (FSs). Whenever the new connection requests for FSs, the reactive approach check for the suitable FS, if it's not accessible, the connection will be blocked. Therefore, both approaches improvise the performance of the network. The reconfiguration of the network can be split into two categories without re-routing and re-routing. The re-routing method assigned the new route and spectrum resources respectively for existing established connections that may cause traffic disruption. Without re-routing, the resources of the spectrum can be changed along with the same route without any traffic disruption. Figure 3 shows how to align the FSs after each termination of a connection from the network for future demands.

\section{PROPOSED APPROACH}

The Jellyfish Search Optimization (JSO) technique is eminent for cracking the complicated problems in several domains. This technique is evolved from the behavior of jellyfish in the ocean. It is known as Artificial Jellyfish Search Optimizer. Jelly Fish survive under the water with different environmental conditions. Jellyfish resemble a bell shape and it has different varieties in terms of physical features such as colors, shape, and size. Jellyfish are grouped and make a Jelly Fish swarm when the environmental conditions favor the jellyfish and a huge amount of jellyfish formation called Jellyfish bloom. When the temperature of the water increases, more oxygen, plenty amount of nutrients, and water that has more iodine is suited to jellyfish for surviving and its productions. It traps their foods by using tentacles and their stings [14]. The proposed work is carried out by deploying the jellyfish search optimization technique in a proactive and reactive defragmentation approach as PR-DF-JSO.

Whenever a new connection request arrives in the network the reactive defragmentation invokes and allocates the spectrum resources (FSs) based on the need. After terminating each connection from the network the proactive algorithm invokes and rearranging the spectrum slots for upcoming connection requests. The number of reallocation of connections done is directly proportional to the increase in the usage of the spectrum as well as network resources. Instead of traversing all the connections to reallocate, traverse only the most required connection. Here, the Jellyfish search optimizer is used to traverse the connection to reallocate. Jellyfish migrates to new ocean currents periodically is one of its behavior. Similarly, whenever a new connection request arrives, then the jellyfish search optimizer moved to the latest requests connected to the network and that connection alone can reallocate to some other path of the network. Once the connection is reallocated again jellyfish sticks to the connection which has recently connected to the network path without any disruption to other paths or the connections in the network and during the reallocation of a connection, rerouting is possible. Re-routing the path is achieved based using RSA and its constraints were implemented for better defragmentation of the network spectrum. This is being done while there is a heavy network traffic load which is related to the formation of a jellyfish swarm. Henceforth, it improves the network resource utilization and minimizing the bandwidth blocking probability compare to the prevailing approaches.

Let us consider the network $\mathbf{N}(\mathbf{n}, \mathbf{L}, \mathbf{T})$ where $\mathrm{n}$ represents the number of nodes present in the network, $\mathrm{L}$ represents the number of optical fiber links in the network, $\mathrm{T}$ represents the cost of the distance of each node present in the network in Kilometer. Every fiber links have a number FSs. A new connection request from one node to another node ie., Source $\mathrm{S}$ to destination $\mathrm{D}$ represents in equ 1. 


$$
\mathbf{R}_{\mathbf{S}, \mathbf{D}}=\left(\mathbf{A s}_{\mathbf{S}, \mathbf{D}}, \mathbf{H}_{\mathbf{S}, \mathbf{D}}, \mathbf{F S s}\right)
$$

where the random variable $\mathbf{A}_{\mathbf{S}, \mathbf{D}}$ represents the arrival rate is assumed as poison with mean $\overline{\boldsymbol{A}_{\boldsymbol{S}, \boldsymbol{D}}}, \mathbf{H}_{\mathbf{S}, \mathbf{D}}$ represents the holding time of each request as an exponential distribution $\overline{\boldsymbol{H}_{S, \boldsymbol{D}}}$ and FSs represents the number of spectrum slots needed for each connection request. This may vary in the different route of a single request based on the distance and modulation format. The FSs are calculated as per the equ 2.

$$
\mathbf{F S s}=\left(\left[\mathbf{B}_{\mathbf{S}, \mathbf{D}} / \text { Ceon-mod }\right] \mathbf{H}_{\mathbf{S}, \mathbf{D}}\right)+\mathbf{G B}
$$

where Bs,D is the essential bit rate of each request in Gbps, Ceon-mod denotes the slot volume consistent modulation format and GB is the Guard band. Here the modulation format varies (bits/symbol) based on the optical reach distance [15].

The current available FSs index of each link in the network is calculated using equ 3.

$$
\mathbf{C F}_{1}=\sum_{\mathrm{i}=0}^{\mathrm{f}-1}\left|\mathrm{~F}_{\mathrm{l}, \mathrm{i}}-\mathrm{F}_{\mathrm{l}, \mathrm{i}+1}\right|
$$

where $\mathbf{F}_{1, \mathrm{i}}$ is defined as a binary variable which is treated as 1 when the $\mathrm{FS}_{\mathrm{i}}$ is already used or occupied in the link 1 of the path $\mathrm{P}_{\mathrm{i}}$, and otherwise it is represented as 0. The range FSs needed for each connection demand varies between 1 to 16 FSs depending on the connection bit rate and supported speed also may differ from $10 \mathrm{Gbps}$ to $1 \mathrm{~TB} / \mathrm{s}$.

The jellyfish search optimizer is based on the three conditions. 1) Jellyfish either follow the sea momentum or move inside the crowd. 2) Jellyfish move in the sea looking for food. They are more pulled in to areas where the accessible amount of food is more significant. 3) The amount of food discovered is resolute by the location and it is matched to the objective function.

The movement of jellyfish around their location and compared with refreshed location of every jellyfish are given in equ 4.

$$
J_{i(t+1)}=J_{i(t)}+\gamma \times \operatorname{random}(0,1) \times(\text { Upper }- \text { Lower })
$$
locations.

Where $\gamma$ is a motion constant that is associated with the distance of the movement of jellyfish

The boundary constraint for jellyfish movement in the network is defined as equ 5.when the jellyfish moved outside the boundary constraint it will return the opposite bound.

$$
\begin{aligned}
J_{i, d}^{\prime} & =\left(J_{i, d}-\text { Upper }_{d}\right)+\text { Lower }_{d}(\text { d }) \text { if } J_{i, d}>\text { Upper }_{d} \\
J_{i, d}^{\prime} & =\left(J_{i, d}-\text { Lower }_{d}\right)+\operatorname{Upper}_{d}(\text { d }) \text { if } J_{i, d}<\text { Upper }_{d}
\end{aligned}
$$

Where $\boldsymbol{J}_{\boldsymbol{i}, \boldsymbol{d}}$ represent the present location of $\mathrm{i}^{\text {th }}$ jellyfish, $\boldsymbol{J}_{\boldsymbol{i} \boldsymbol{i} \boldsymbol{d}}$ is the updated location after checking the boundary constraints. $\boldsymbol{U p p e r}_{\boldsymbol{d}}$ and Lower $_{\boldsymbol{d}}$ are upper links and lower links of $\mathrm{d}^{\text {th }}$ element in the search phase.

The proposed work aim to minimize the blocking probability, increase the spectrum utilization without any traffic disruption. The hitless fragmentation algorithms are worked without any re-routing the connection path for the reason to avoid network disruption but its chances to increase the number of connection blocks $[16,17]$. For example, peak hour's heavy traffic situations cannot establish the connection for the entire request. It allows only a minimal amount of requests to the network so; the neediness of the defragmentation algorithm should flexible for all kinds of traffic demand.

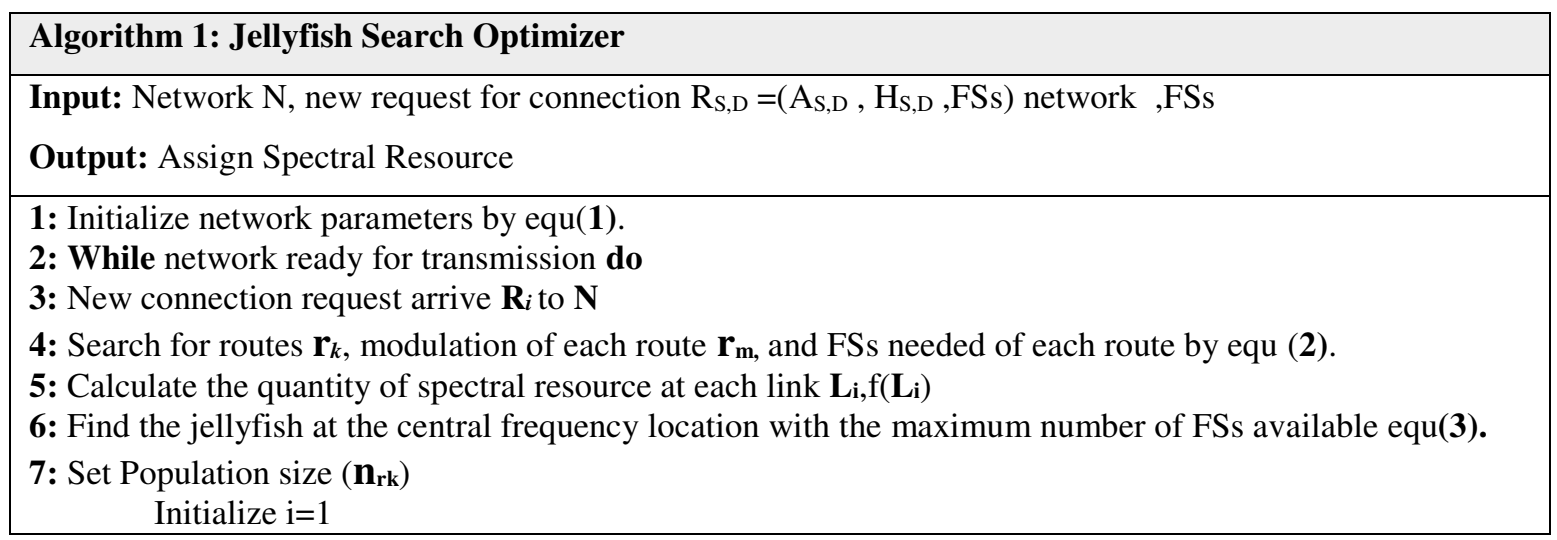




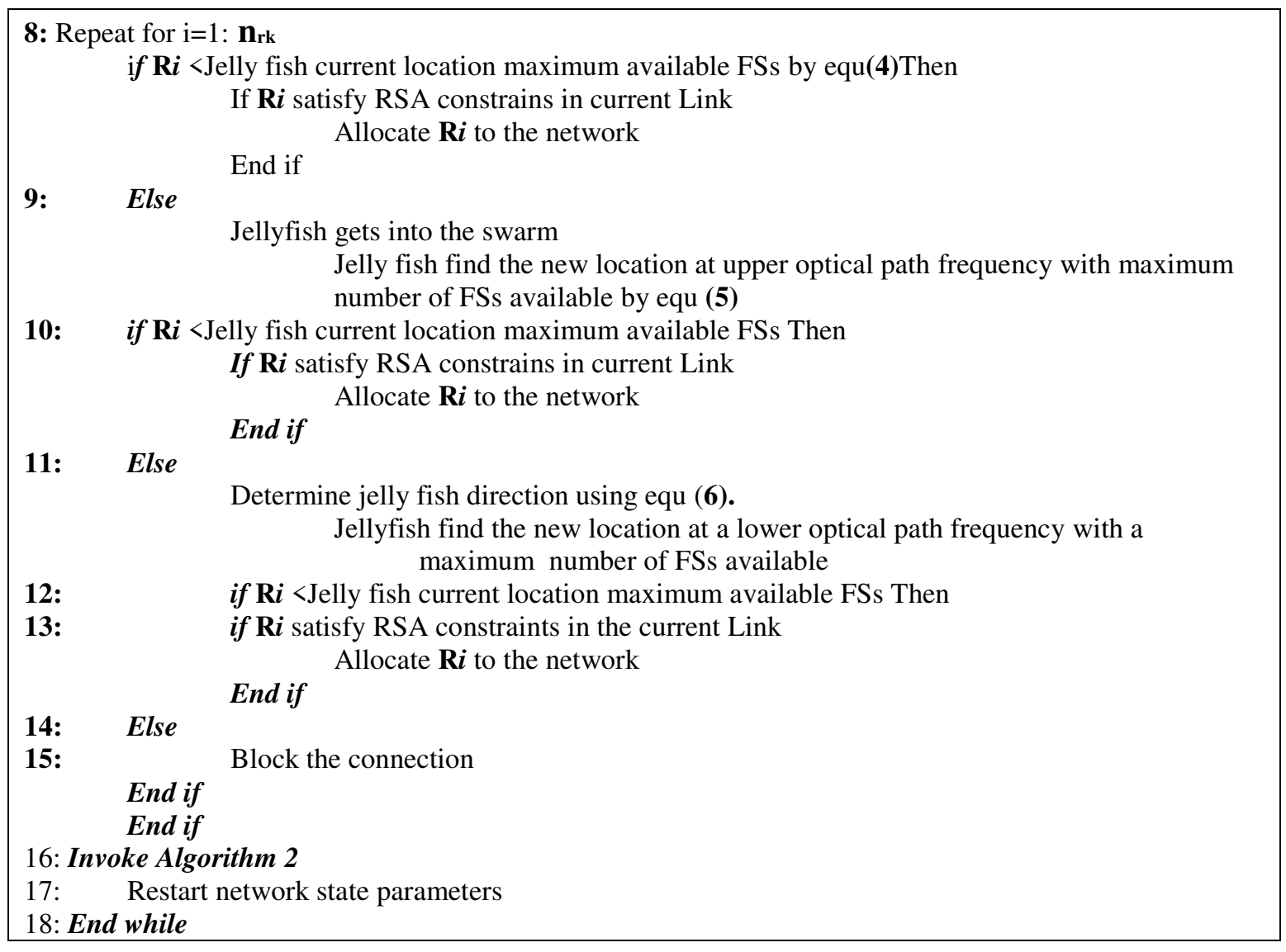

Algorithm 1 presents overall working defragmentation in EON. In this algorithm when the new request reach the network before the light path admits to the transmission, the initial works like construct the routing table, number of FSs needed for all possible paths between the source and destination, and guard band are to be completed. The guard band may be varying depends upon the travel distance and amount demand with respective modulation format.

The algorithm first finds the central frequency of each $\mathrm{i}^{\text {th }}$ route path if the number of FSs available with RSA constraints then it admits establishing the connection. If the central frequency light path is not available for establishing connection then change location upper or lower optical path of the link simultaneously of all possible routes in the routing table. After blocking the connection again the algorithm 2 is invoke and check all free FSs in each link possible to reassign the same link otherwise reroute the connection. Algorithm 2 ensures the availability of more contiguous FSs for upcoming demands.

\section{Algorithm 2: Reassign FSs and reconfigure the network}

Input: Current state of the network, $\mathbf{K}$ number of active connections present in the network, $\mathbf{T}$ total occupied FSs in each link in the network, MinF is the minimum FSs contiguous slot assigned and MaxF is the highest FSs slot assigned in each connection, all routes in routing table $\mathbf{r} \mathbf{i}, \mathbf{L}$ total links of the network.

Output: New State of the network

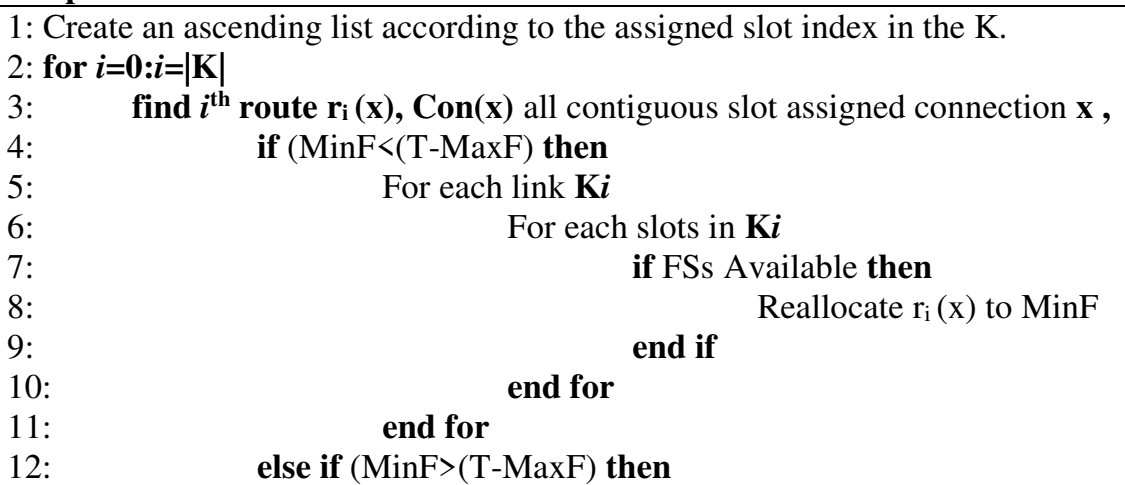




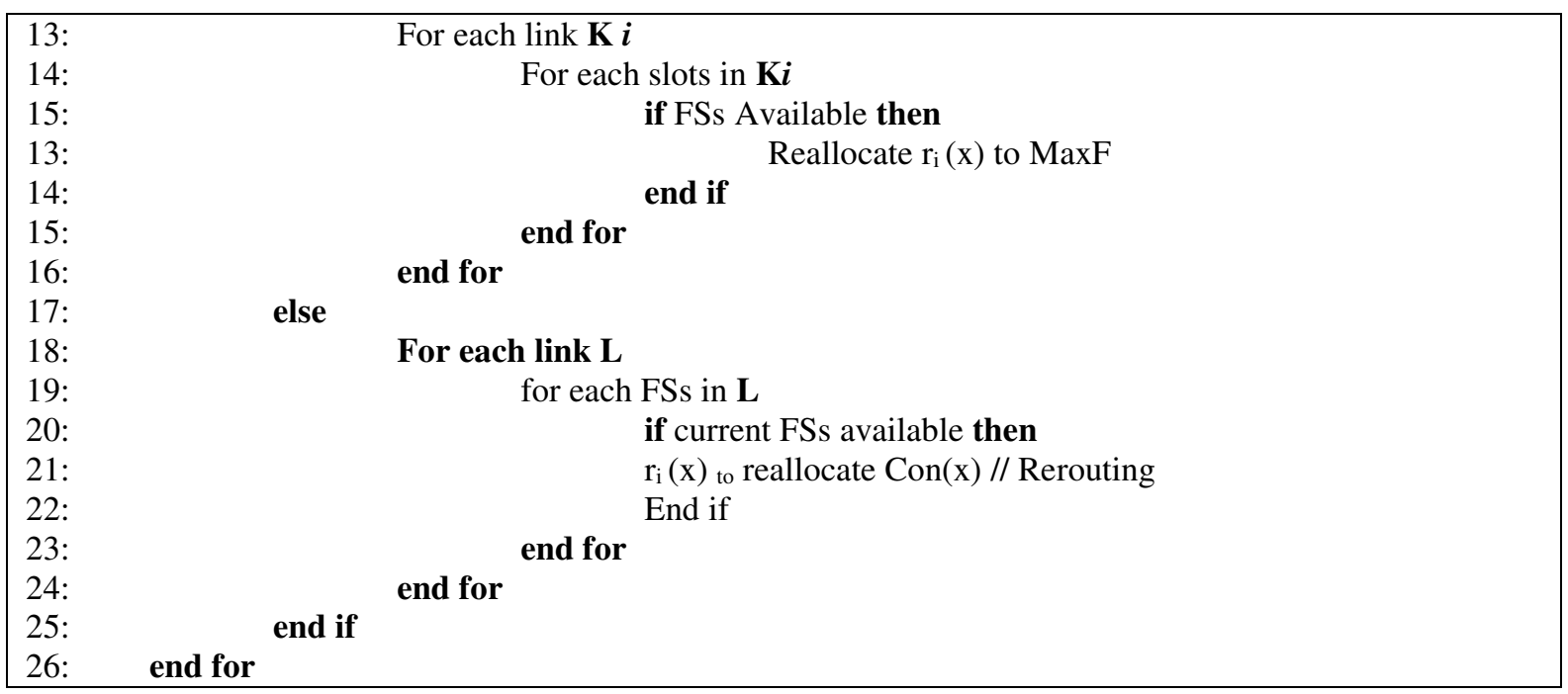

The connections are reorganized the initial and final stage of spectrum allocation if it ensures the more availability of spectral resources for upcoming demands. The rearranging of spectrum slots hitless method and rerouting is complete by make-before-break to avoid network disruption.

\section{RESULTS AND DISCUSSION}

This work fully focused on minimizing the disruption and reconfiguration rate of the EON and utilizing the overall spectrum to its fullest. This paper combines three approaches to attain the expected output. The overall working mechanisms of the techniques are discussed briefly in the previous section. This section focus on testing the significance of the system and comparing the values with the existing state-of-the-art spectrum defragmentation techniques. Three standard quality metrics were considered to achieve the overall performance of the system under various scenarios. They are Blocking Probability /Blocking Ratio, Bandwidth Fragmentation Ratio, and Spectrum Utilization Gain (SUG).

The 14 nodes and 21 links The National Science Foundation Network (NSFNET)[18] and 24 node 43 USNET [19] optical network topologies are used for the simulation purpose. The simulations are made by MATLAB tool and bandwidth of each fiber links is $1 \mathrm{THz}$. The subcarrier slot intervals are $12.5 \mathrm{GHz}$ so each fiber link has 320 FSs. All the demand arrivals are poisson process arrival rate $\lambda$ on average with a lifetime of each arrival measured by Erlang as per [8].

The main performance analysis metric of the algorithm is Bandwidth Blocking Probability (BBP) or Blocking Probability (BP) used to calculate the disruption rate in the EON. Every time when the system reconfigures the connection, the existing connections are disrupted and leads to poor quality of service. In simple terms, it is the ratio between the numbers of blocked connections to the overall connections request. If the results of the BP are smaller, then the fragmentation rate also tends to be smaller as per the equ 7 . The formula to calculate BP is equated below [20].

$$
B P=\frac{\text { Numberof connectionsblocked }}{\text { Total volume traffic offered to the network }}=\frac{\sum B C}{\sum C_{b}}
$$

Bandwidth Fragmentation Ratio (BFR) denotes the number of non-aligned, isolated, and noncontiguous slots in EON among the contiguous, allocated, and connected FSs. This ratio shows the current fragmentation status of the network at any given point of time. If BFR is high, then the BP is also more likely to be high. The proposed hybrid defragmentation technique effectively tracks and handles the BFR by defragmenting and reconfiguring the FSs links which will not affect the overall QoS of the spectrum. The below equation 8 is used to calculate the BFR of any given link in the network [18].

$$
\tau_{l}=\left\{\begin{array}{c}
1-\frac{\operatorname{MassBlk}\left(F S_{l}\right)}{N-\sum\left(F S_{l}\right)} \text { if } \sum\left(F S_{l}\right)<N, \\
\text { 0if } \sum\left(F S_{l}\right)=N
\end{array}\right.
$$

Where $\operatorname{MassBlk}\left(\boldsymbol{F} \boldsymbol{S}_{l}\right)$ is the size of the current massive block in $l, \mathrm{~N}$ represents number of frequency slots per link and $\sum\left(F S_{l}\right)$ indicates the sum of allocated frequency slots in link $l$.The formula to find the BFR for the entire network which is denoted as $F_{R}$ is, 


$$
F_{R}=\frac{\sum_{\forall l \epsilon L} \tau_{l}}{|L|}
$$

In the above equation, $\mathrm{L}$ indicates the group of links and $|L|$ represents the number of links in EON. The Spectrum Utilization Gain (SUG) value indicates how much the spectrums are utilized its bandwidth without rejecting numerous connections unnecessarily due to poor FSs allocation caused by fragmentation [18]. Defragmentation also plays a vital role in SU, by effectively managing and balancing the reconfiguration and disruption rate deprived of compromising the overall throughput of the network spectrum. SU depends on Bandwidth, Space, and \& Time factors.

$$
\boldsymbol{S U}=\boldsymbol{B} * \boldsymbol{S} * \boldsymbol{T}
$$

SUG can be calculated as per equ 11 ,

$$
\boldsymbol{S U G}=\frac{G}{S U}
$$

Where $\mathrm{G}$ is the Gain obtained after defragmented slots in the network. The evaluation is done using various network traffic densities under which the quality metrics are tested. The blocking probability results are shown in table 1 and 2 with various network loads (Erlang).

\begin{tabular}{|c|c|c|c|c|c|c|}
\hline Erlang & FA-RSA & HTAR & HOPS & PRDEF & D-Gen40 & PRDFJSO \\
\hline $\mathbf{1 0 0}$ & 0 & 0 & 0 & 0 & 0 & $\mathbf{0}$ \\
\hline $\mathbf{2 0 0}$ & 1.26 & 3.25 & 2.25 & 1.0 & 0.9 & $\mathbf{0 . 9}$ \\
\hline $\mathbf{3 0 0}$ & 9.21 & 7.21 & 5.92 & 4.65 & 3.25 & $\mathbf{3 . 1 5}$ \\
\hline $\mathbf{4 0 0}$ & 15.25 & 14.23 & 13.1 & 11.15 & 10.9 & $\mathbf{1 0 . 2 5}$ \\
\hline $\mathbf{5 0 0}$ & 22.18 & 20.85 & 19.56 & 18.24 & 14.7 & $\mathbf{1 3 . 9 5}$ \\
\hline $\mathbf{6 0 0}$ & 26.18 & 23.63 & 22.35 & 21.14 & 21.7 & $\mathbf{2 0 . 0 9}$ \\
\hline
\end{tabular}

Table 1. Blocking Probability (\%) results in different traffic loads of NSFNET.

The bandwidth blocking probability is defined as the ratio of number of connection rejected to the total number of connection requested [21]. When the bandwidth blocking probability is lower than the network the transmission rate are increases, this works aims to reduce the bandwidth blocking probability ratio with different traffic load. First evaluate the algorithm with NSFNET topology that progressively increases the traffic load between 100 to 600 (Erlang) results compared with existing fragmentation algorithms and similarly for USNET topology.

\begin{tabular}{|c|c|c|c|c|c|c|}
\hline Erlang & FA-RSA & HTAR & HOPS & PRDEF & D-Gen40 & PRDFJSO \\
\hline $\mathbf{1 0 0}$ & 0 & 0 & 0 & 0 & 0 & $\mathbf{0}$ \\
\hline $\mathbf{2 0 0}$ & 1.56 & 3.1 & 2.1 & 0.9 & $\mathbf{0 . 7 5}$ & 0.8 \\
\hline $\mathbf{3 0 0}$ & 10.15 & 7.45 & 6.5 & 4.76 & 3.50 & 3.55 \\
\hline $\mathbf{4 0 0}$ & 16.35 & 15.23 & 13.25 & 11.25 & 9.89 & $\mathbf{9 . 7 5}$ \\
\hline $\mathbf{5 0 0}$ & 23.50 & 21.25 & 21.5 & 17.9 & 14.8 & $\mathbf{1 4 . 7 5}$ \\
\hline $\mathbf{6 0 0}$ & 26.75 & 24.1 & 23.45 & 22.7 & 22.3 & $\mathbf{2 1 . 7}$ \\
\hline
\end{tabular}

Table 2. Blocking Probability (\%) results in different traffic loads of USNET. 


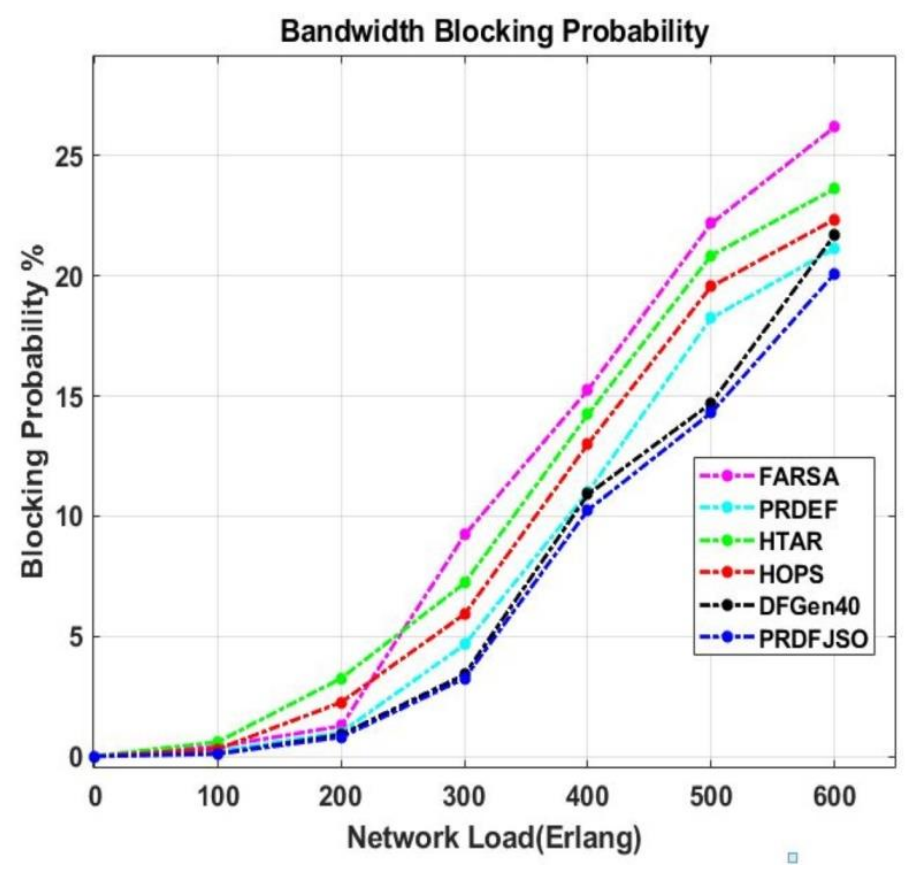

Figure 4. Bandwidth blocking Probability of different traffic loads in NSFNET.

The Blocking Probability (BP) results shown in figure 4 and 5, which represents the proposed algorithm rejection rate is less compared to other fragmentation algorithms. When traffic load is very low 100 to 200 Erlang proactive algorithms are works with very less blocking rate. Conversely, the traffic load increase HTAR and HOPS improves the performance compared to proactive algorithm FARSA. The algorithms PRDEF and DFGen40 worked in both conditions with less number of blocks in the connection. In the entire scenario the proposed algorithm works with a very less number of blocks. It denotes that, under various heavy traffic loads, the proposed system defragments the spectrum efficiently without compromising the QoS. From the above illustration, it is clear when the traffic load is low, then the proactive algorithms like H-HT and H-PP outperformed the reactive ones. In contrast with that, reactive algorithms like RHOPS and HTAR outperformed proactive algorithms. The average Blocking Probability (BP) of PRDFJSO in NSFNET is $8.06 \%$ compared with existing algorithm D-Gen40 8.58\% and also PRDFJSO is lower percentage of Blocking Probability (BP) all other fragmentation algorithms. Similarly for USNET the PRDFJSO is $8.43 \%$ and D-Gen40 $8.54 \%$ both algorithms are not as much of blocking in the connection request in various traffic situation.

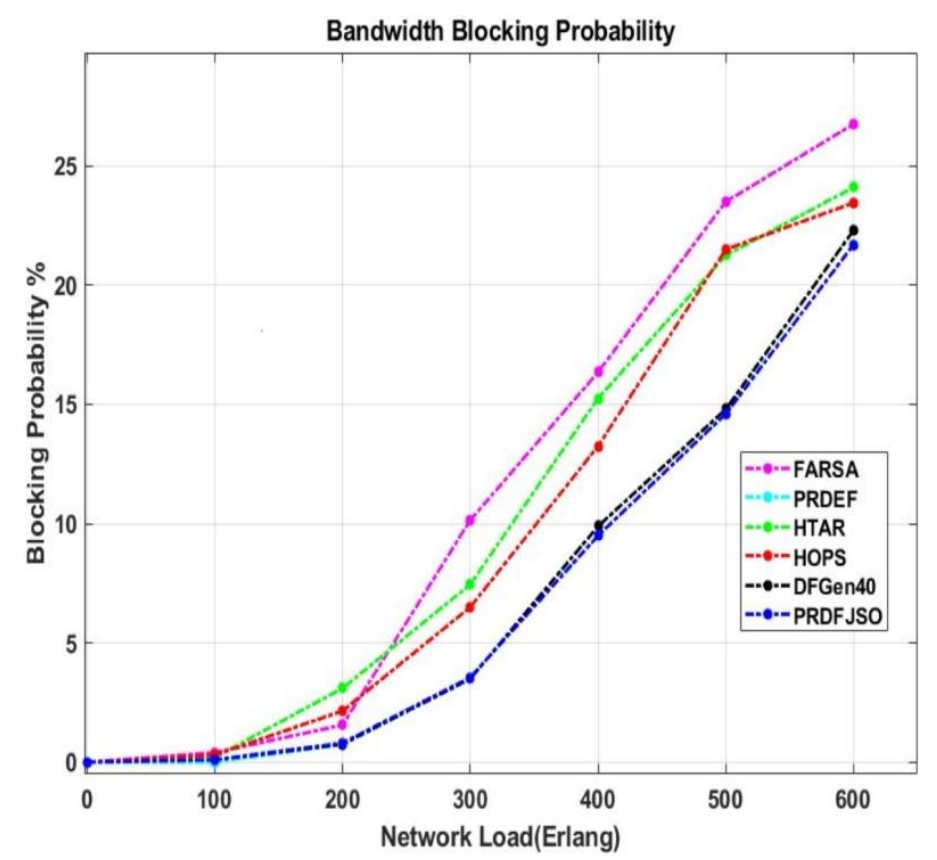

Figure 5. Bandwidth blocking Probability of different traffic loads in USNET. 
The proactive algorithm reconfigures the FSs periodically to avoid fragmentation. It leaves certain slot blocks for future connections. But due to dynamic and heavy traffic loads, the left slot blocks may not be enough for the current network requirement to establish a connection and there are still chances for the new connection to be rejected. The reactive algorithms are invoked if there are new connection requests arises. The reactive algorithm reconfigures the FSs based on the requirement thus reduces the number of rejected connection requests. There is still a problem of reconfiguring many FSs and interrupts the available connection which led to poor QoS and the disconnected connection have more chance to re-connect which in turn increases the traffic load unnecessarily.

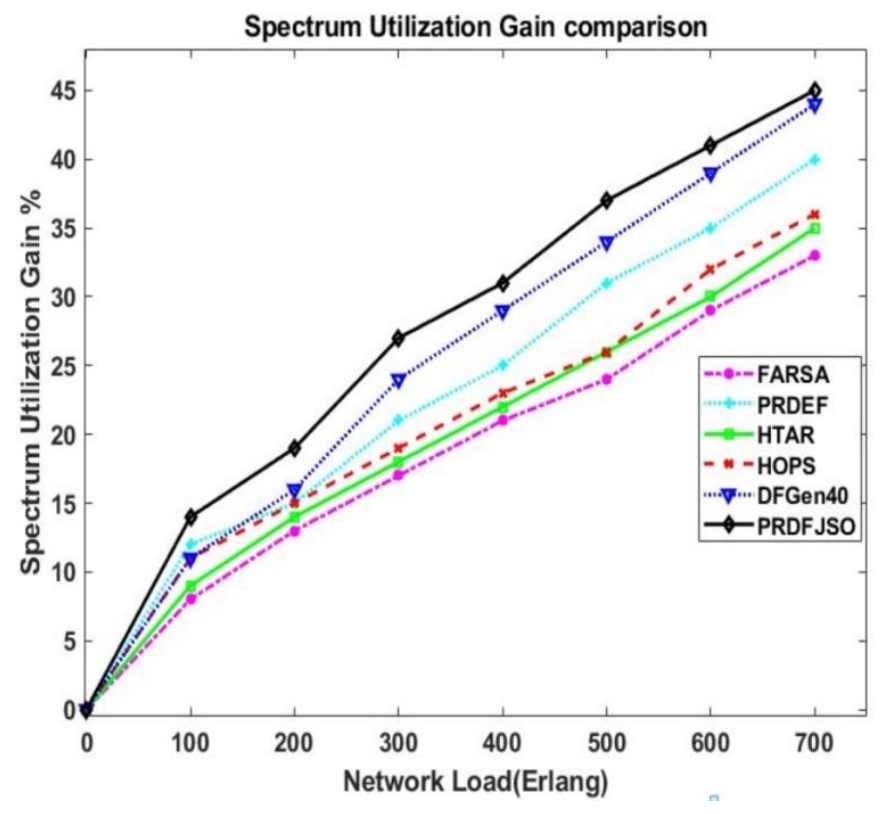

Figure 6. The Spectrum Utilization Gain Comparison in NSFNET.

The Spectrum Utilization Gain (SUG) exposes the overall progressive impact of the proposed system on the EON spectrum. If SUG is high, also other metrics performed up to the expectancy level. The proposed algorithm is achieved more spectrum gain compared with other algorithms.

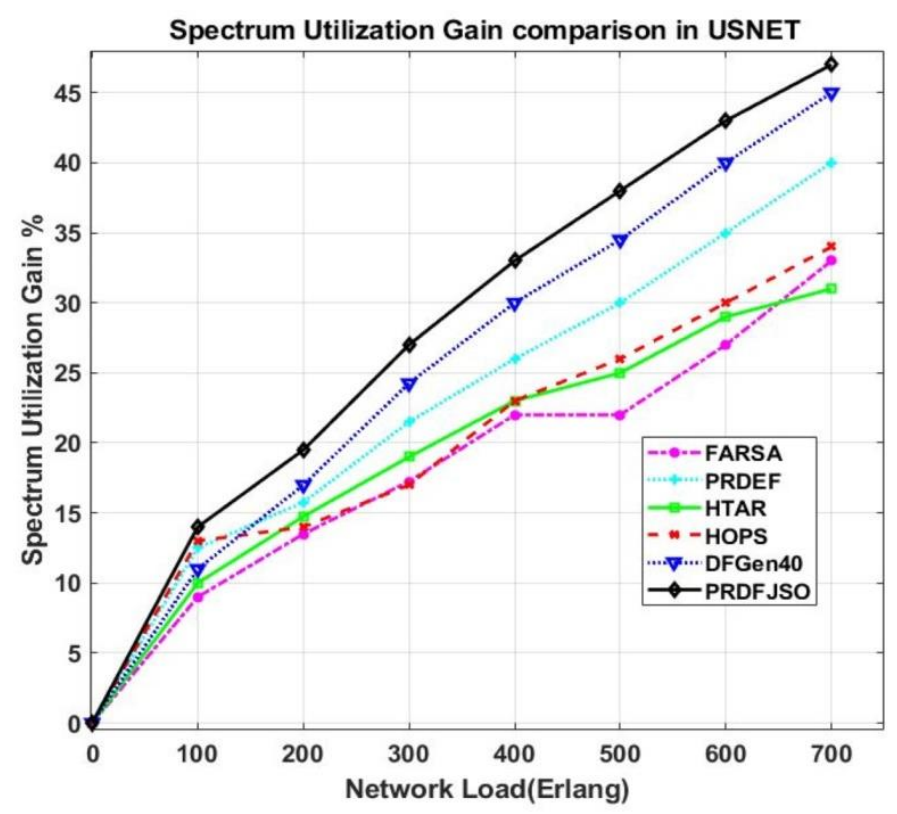

Figure 7. The Spectrum Utilization Gain Comparison in USNET.

Figures 6 and 7 shows the spectrum utilization gain with different network load PRDEF and DFGen40 utilizes more spectrum compared to other fragmentation algorithms; however, both PRDEF and DFGen40 algorithms realign the FSs before and after assigns the FSs for every cycle of the connection request. It increases 
the transmission rate and produces efficient utilization of the spectrum. Similarly, the proposed algorithm works in the same principle which utilizes more spectrum FSs compared to other existing algorithms.

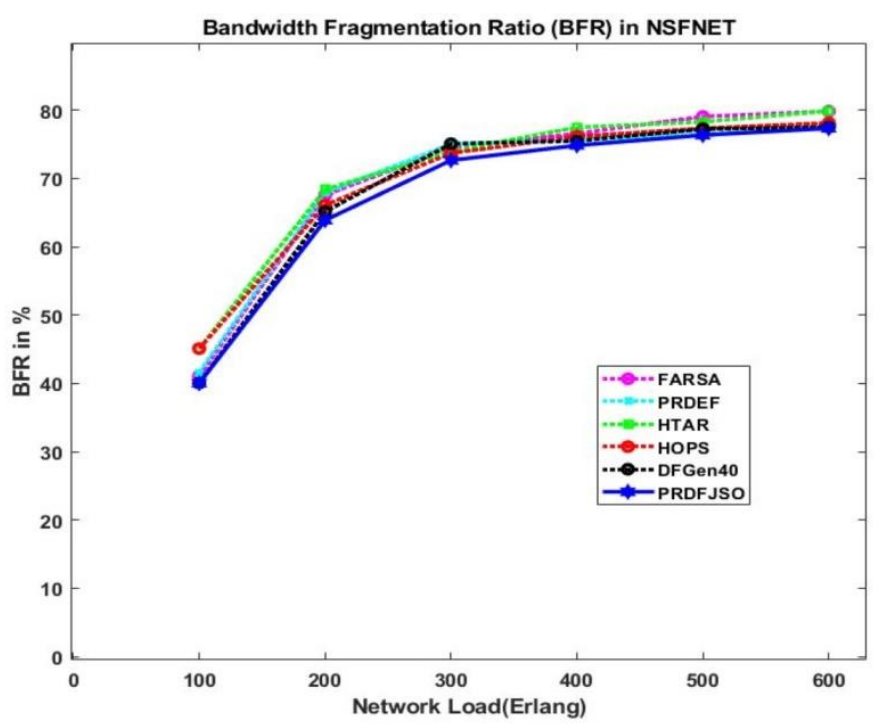

Figure 8. Bandwidth Fragmentation Ratio (BFR) Comparison in NSFNET

The figure 8 shows the bandwidth fragmentation ratio (BFR) with different traffic load (Erlang) in NSFNET. From the experimental observation the proactive algorithms are performed better than the reactive algorithms. However the impact of bandwidth fragmentation ratio is not speculate performance of blocking probability of the defragmentation algorithms. The above illustration exhibits the constructive impact of the proposed system on the network by gradually reducing the BFR by allocating the isolated slots in the spectrum and makes it usable for future connection requests. Similarly the results of BFR in USNET with traffic load (Erlang) shows in Figure 9. So the blocking of the connection may happen not only for defragmentation issue, it may also be other reasons like high holding time of connection request and limited in resources.

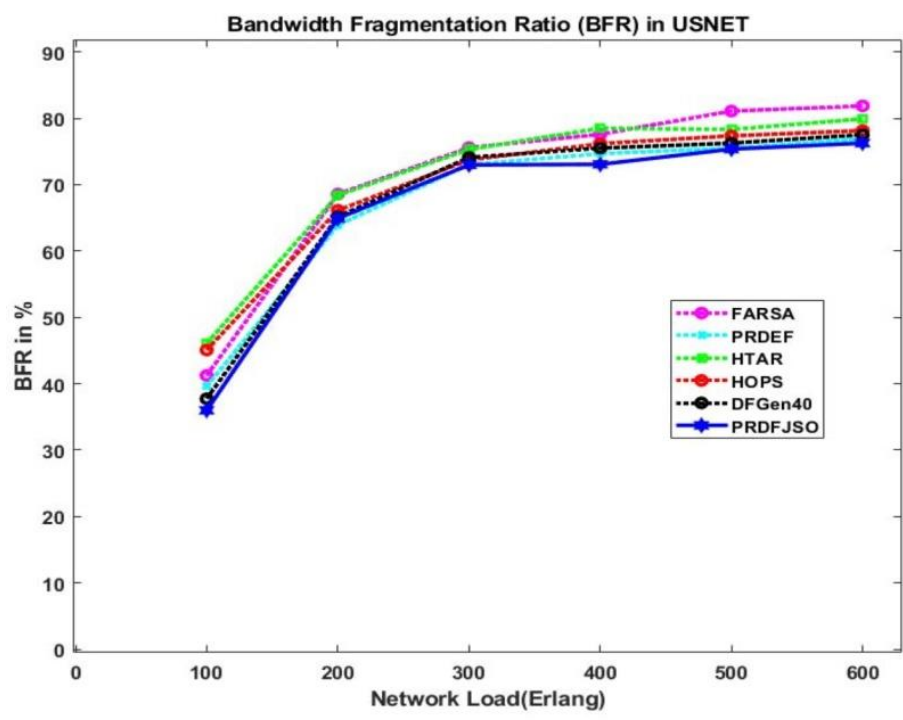

Figure 9. Bandwidth Fragmentation Ratio (BFR) Comparison in USNET

In addition to the metrics, the reconfiguration rate of the existing connection links in the EON spectrum after each defragmentation is also measured in percentage and the graphical representation is illustrated below. 


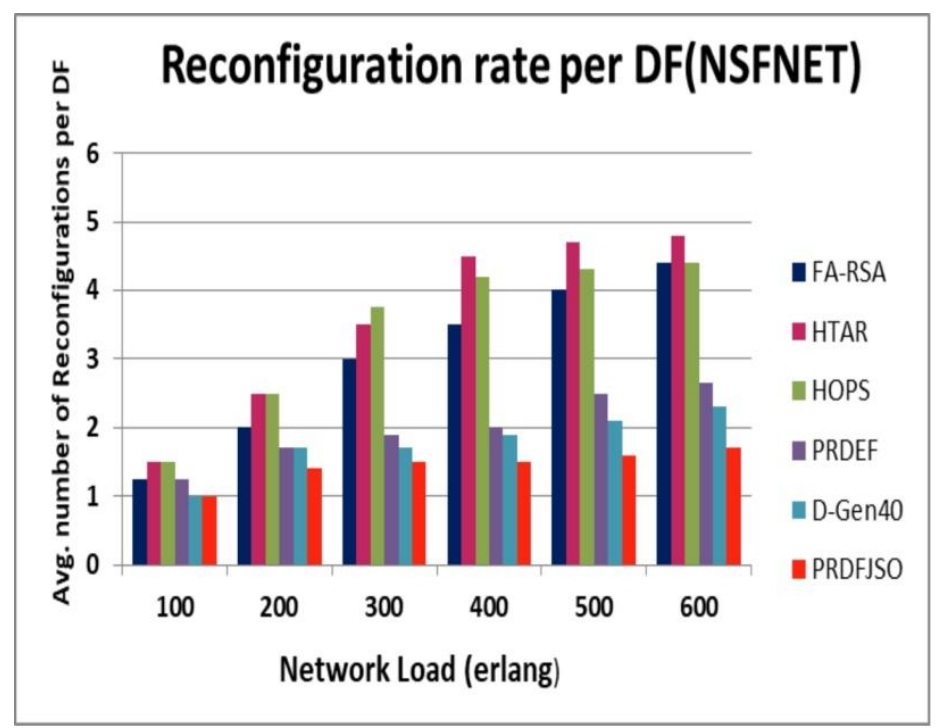

Figure 10. Average number of reconfigured connections for every performed DF (NSFNET).

The figure 10 shows the average numbers of connections are reconfigured in each time defragmentation is performed in NSFNET with different traffic load. The number of reconfiguration of the connection is higher that may disrupt the network traffic. Similarly less reconfiguration rate is achieved when the defragmentation is performed that increase the transmission rate. A higher number of reconfigurations, however often results in lower connections blocking. Note that during reconfiguration, the proactive approach does not affect the ongoing traffic between any connections. Likewise, the number of the reconfiguration is gradually (increases) with the change in load. The proposed algorithm reconfiguration rate increases upto lower load (upto 400 Erlang) but in higher load it maintains reconfiguration rate of connection.

\section{CONCLUSION}

In this paper, various combined techniques are employed to collectively fetch better spectrum utilization in EON. The experimental result shows the significance and importance of the proposed work is outperformed the existing defragmentation algorithms. Under various traffic loads and network circumstances, the proposed work relatively performed well based on the quality metric values. The quality metrics considered for this work are Blocking Probability, Spectrum Utilization Gain, and Bandwidth Fragmentation Ratio. The combination of Proactive and Reactive strategies together leads to a more complex system. But the proposed system uses Jellyfish Search optimization technique is relatively reduces the complexity of FSs index realignment and connection reconfiguration. The proposed system acts effectively under various network loads and scenarios of the EON.

\section{Declarations}

We confirm that the manuscript is the author's original work and the manuscript has not received prior publication and is not under consideration for publication elsewhere. We declare that we shall not submit the paper for publication in any other Journal or Magazine till the decision is made by journal editors.

\section{Funding}

Not applicable

\section{Conflicts of interest/Competing interests}

No potential conflict of interest

Availability of data and material

Not applicable

Code availability

Not applicable 


\section{References}

[1]N. Kitsuwan, P. Pavarangkoon, and A. Nag, "Elastic optical network with spectrum slicing for fragmented bandwidth allocation," Optical Switching and Networking, vol. 38, p. 100583, Sep. 2020.

[2] B. C. Chatterjee and E. Oki, "Performance evaluation of spectrum allocation policies for elastic optical networks," 2015 17th International Conference on Transparent Optical Networks (ICTON), Budapest, 2015, pp. 1-4, doi: 10.1109/ICTON.2015.7193485.

[3]S. S and M. SS, “A Hybrid meta-heuristic Approach for Optimization of Routing and Spectrum Assignment in Elastic Optical Network (EON),” Enterprise Information Systems, pp. 1-24, Jan. 2020.

[4]D. Batham, D. S. Yadav, and S. Prakash, "Least loaded and route fragmentation aware RSA strategies for elastic optical networks," Optical Fiber Technology, vol. 39, pp. 95-108, Dec. 2017.

[5] Y. Xu and Y. Kim, "Dynamic routing and spectrum allocation to minimize fragmentation in elastic optical networks," 2017 20th Conference of Open Innovations Association (FRUCT), St. Petersburg, 2017, pp. 512518, doi: 10.23919/FRUCT.2017.8071356.

[6] P. D. Singh, D. S. Yadav and V. Bhatia, "Defragmentation Based Load Balancing Routing \& Spectrum Assignment (DLBRSA) strategy for elastic optical networks," 2018 IEEE International Conference on Advanced Networks and Telecommunications Systems (ANTS), Indore, India, 2018, pp. 1-6, doi: 10.1109/ANTS.2018.8710079.

[7]J. Comellas, L. Vicario, and G. Junyent, "Proactive defragmentation in elastic optical networks under dynamic load conditions," Photonic Network Communications, vol. 36, no. 1, pp. 26-34, Apr. 2018.

[8]E. J. Davalos, M. F. Romero, S. M. Galeano, D. A. Baez, A. Leiva, and B. Baran, "Spectrum Defragmentation in Elastic Optical Networks: Two Approaches With Metaheuristics," IEEE Access, vol. 7, pp. 119835-119843, 2019.

[9]S. Fernández-Martínez, B. Barán, and D. P. Pinto-Roa, "Spectrum defragmentation algorithms in elastic optical networks," Optical Switching and Networking, vol. 34, pp. 10-22, Nov. 2019.

[10] S. Ba, B. C. Chatterjee, S. Okamoto, N. Yamanaka, A. Fumagalli, and E. Oki, "Route Partitioning Scheme for Elastic Optical Networks With Hitless Defragmentation," Journal of Optical Communications and Networking, vol. 8, no. 6, p. 356, May 2016.

[11]S. K. Singh and A. Jukan, "Efficient Spectrum Defragmentation with Holding-Time Awareness in Elastic Optical Networks," Journal of Optical Communications and Networking, vol. 9, no. 3, p. B78, Mar. 2017.

[12]R. Wang and B. Mukherjee, "Provisioning in Elastic Optical Networks with Non-Disruptive Defragmentation,” Journal of Lightwave Technology, vol. 31, no. 15, pp. 2491-2500, Aug. 2013.

[13]Y. Wang, "A Research on Spectrum Defragmentation Algorithms in Elastic Optical Network," 2019 2nd World Symposium on Communication Engineering (WSCE), Nagoya, Japan, 2019, pp. 78-81, doi: 10.1109/WSCE49000.2019.9041017.

[14]J.-S. Chou and D.-N. Truong, "A novel metaheuristic optimizer inspired by behavior of jellyfish in ocean," Applied Mathematics and Computation, vol. 389, p. 125535, Jan. 2021.

[15]H. Guo, Y. Li, L. Li, and G. Shen, “Adaptive Modulation and Regeneration-Aware Routing and Spectrum Assignment in SBPP-Based Elastic Optical Networks," IEEE Photonics Journal, vol. 9, no. 2, pp. 1-15, Apr. 2017.

[16]X. Wang, I. Kim, Q. Zhang, P. Palacharla, and M. Sekiya, “A hitless defragmentation method for selfoptimizing flexible grid optical networks," in 38th European Conf. and Exhibition on Optical Communication, Amsterdam, 2012.

[17]F. Cugini et al., "Push-Pull Defragmentation Without Traffic Disruption in Flexible Grid Optical Networks,” Journal of Lightwave Technology, vol. 31, no. 1, pp. 125-133, Jan. 2013.

[18]Xiang Zhou, Wei Lu, Long Gong and Zuqing Zhu, "Dynamic RMSA in elastic optical networks with an adaptive genetic algorithm," 2012 IEEE Global Communications Conference (GLOBECOM), Anaheim, CA, 2012, pp. 2912-2917, doi: 10.1109/GLOCOM.2012.6503559.

[19]R. Vilalta, R. Muñoz, R. Casellas, and R. Martínez, "Virtual opticalnetwork resource allocation using PCE global concurrent optimization for dynamic deployment of virtual GMPLS-controlled WSON," IEEE/OSA J. Opt. Commun. Netw., vol. 5, no. 12, pp. 13731381, Dec. 2013. 
[20]M. Quagliotti, D. C. Arango, M. Schiano, A. Carena, M. Cantono and V. Curri, "Spectrum fragmentation metrics and their use in optical channel allocation algorithms," 19th Italian National Conference on Photonic Technologies (Fotonica 2017), Padua, 2017, pp. 1-4, doi: 10.1049/cp.2017.0187.

[21]D. S. Yadav, A. Chakraborty, and B. S. Manoj, "A Multi-Backup Path Protection scheme for survivability in Elastic Optical Networks," Optical Fiber Technology, vol. 30, pp. 167-175, Jul. 2016, doi: 10.1016/j.yofte.2016.05.003. 
Figures

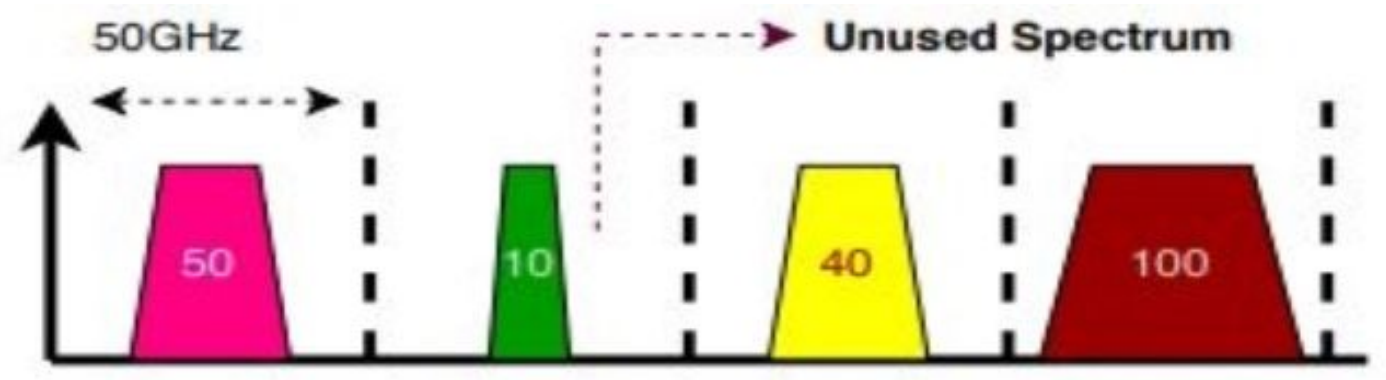

Fixed Grid

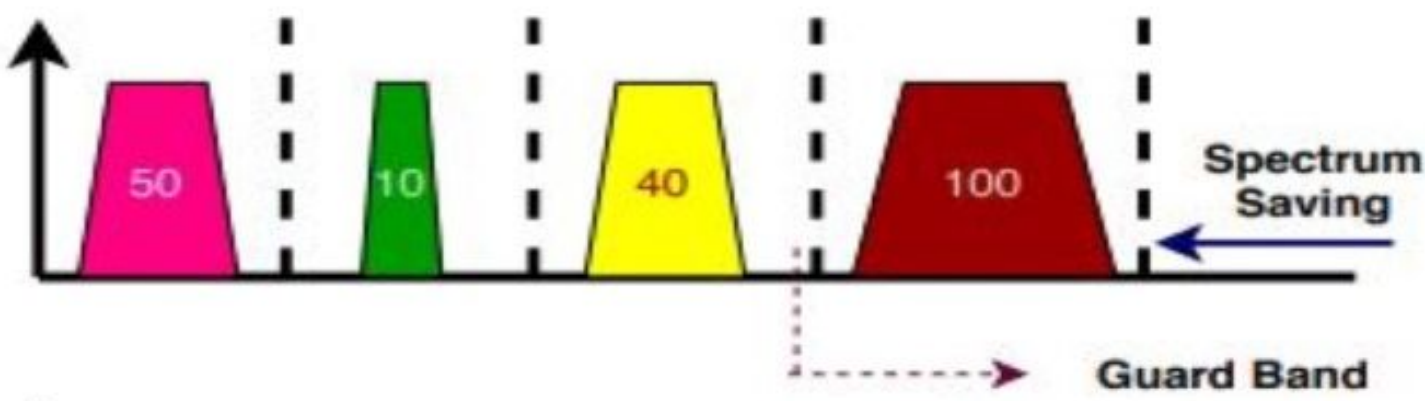

Flex Grid

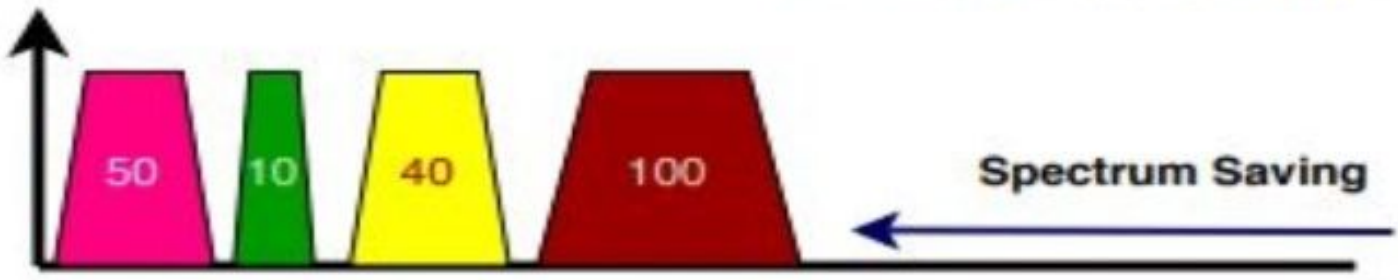

Grid less

Figure 1

Difference between fixed, flex, and gridless network. 

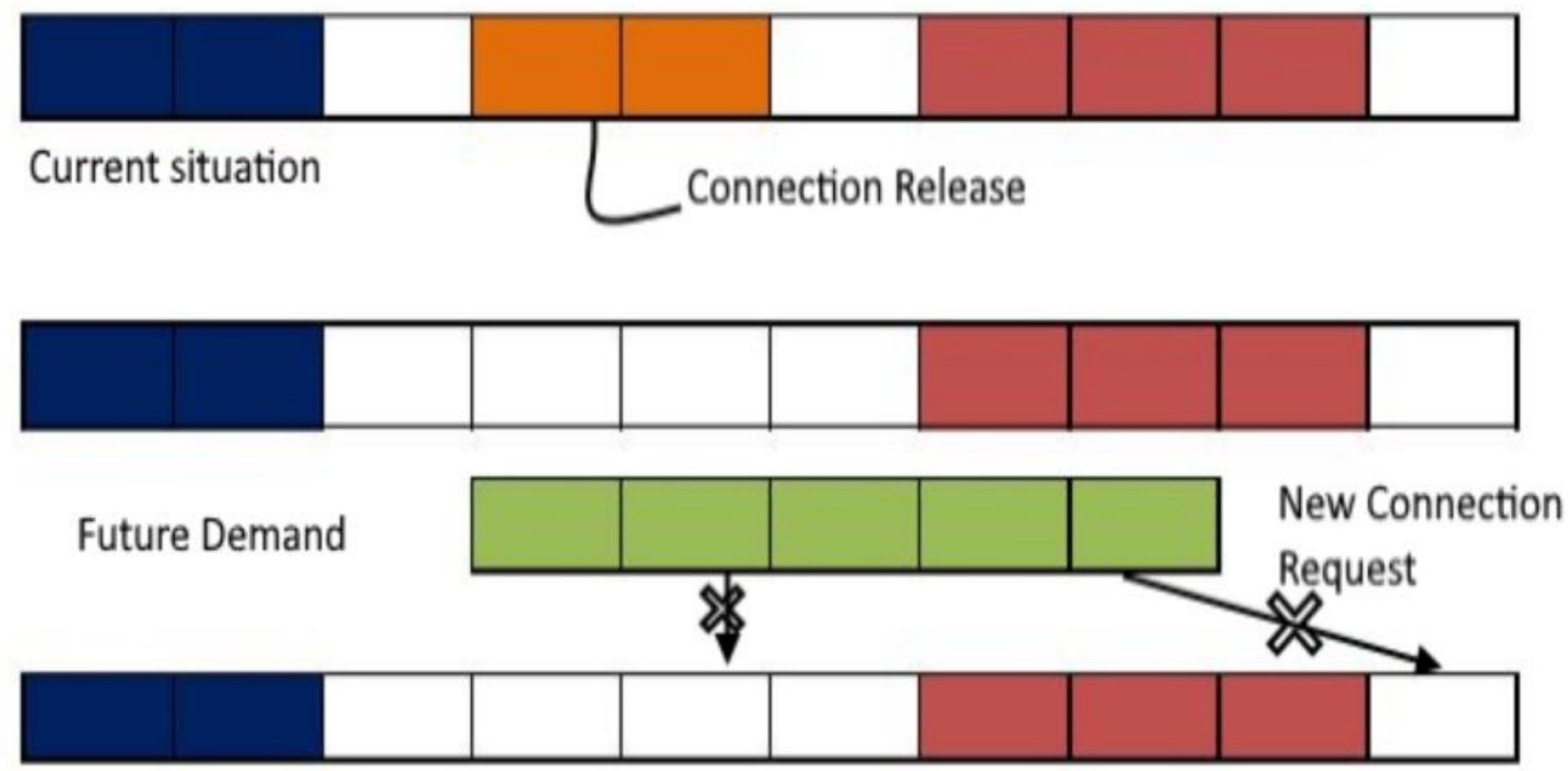

Figure 2

The Spectrum Fragmentation Problem in EON.
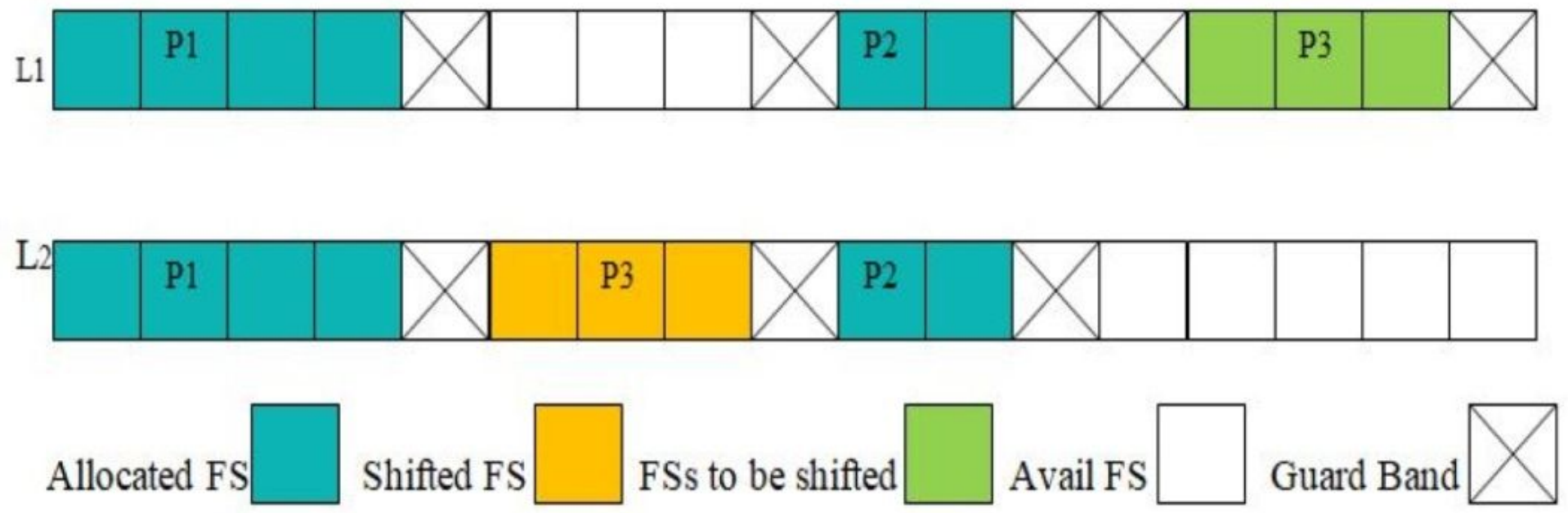

Figure 3

The Spectrum Defragmentation in EON 


\section{Bandwidth Blocking Probability}

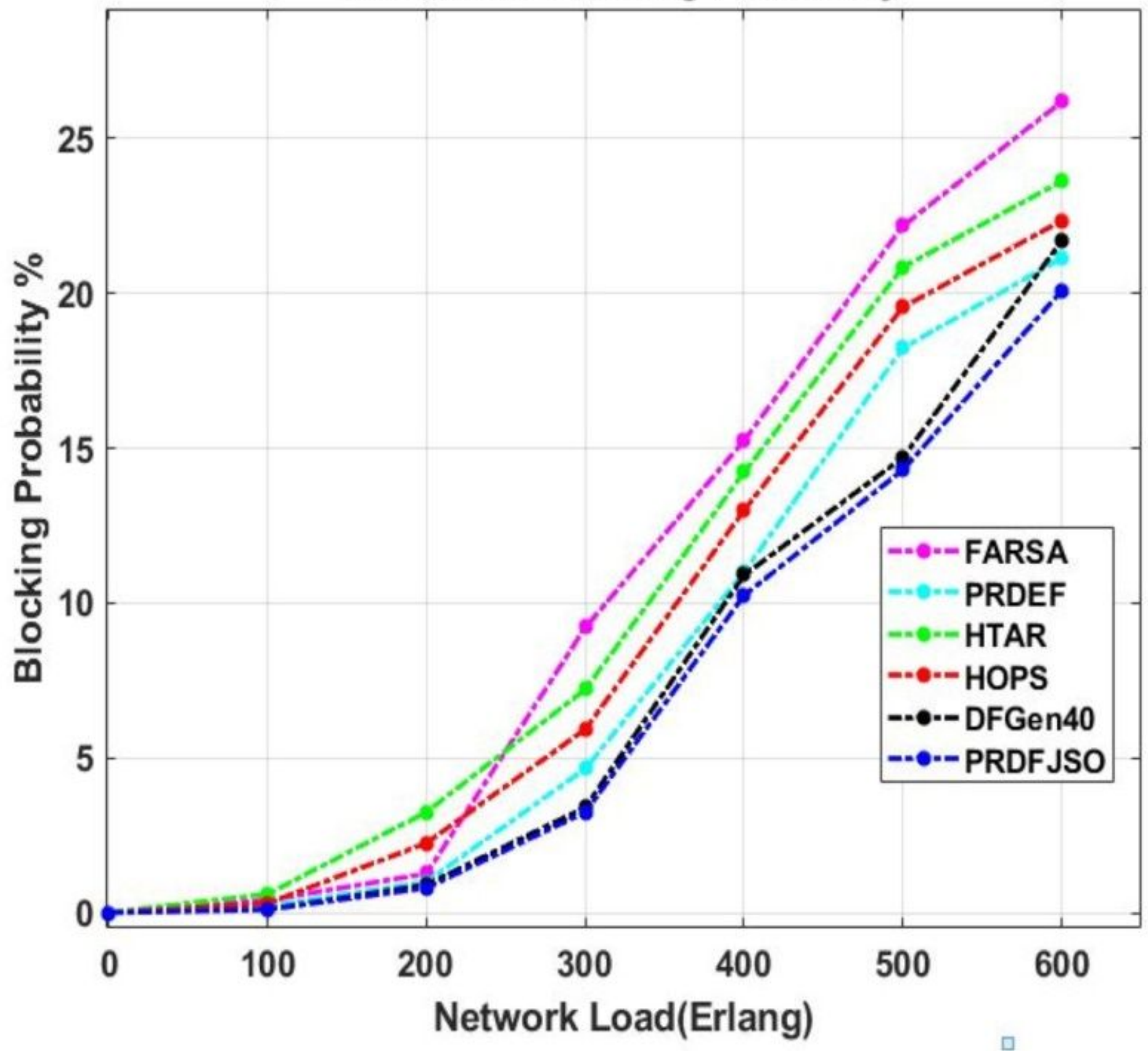

Figure 4

Bandwidth blocking Probability of different traffic loads in NSFNET. 


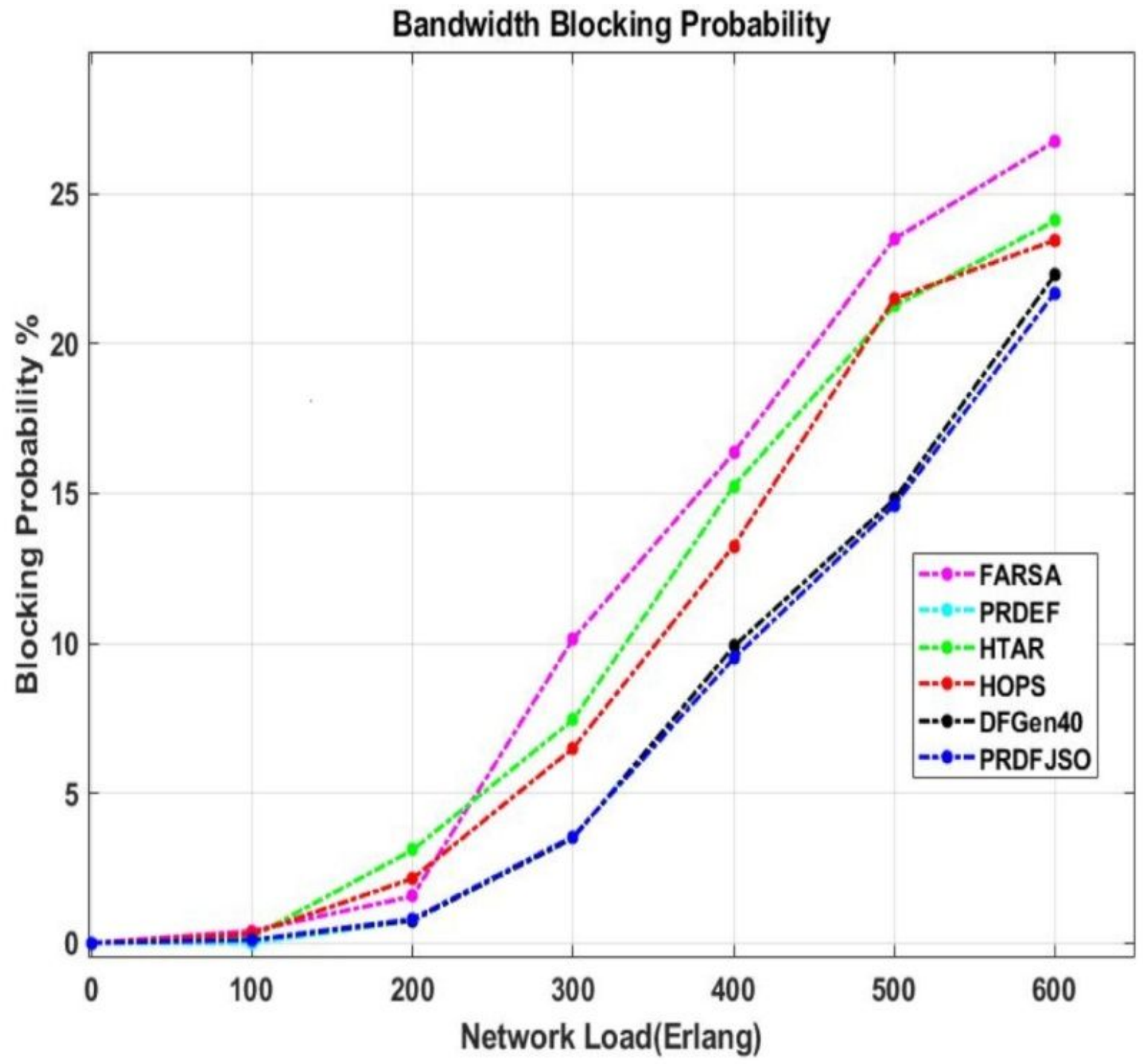

Figure 5

Bandwidth blocking Probability of different traffic loads in USNET. 


\section{Spectrum Utilization Gain comparison}

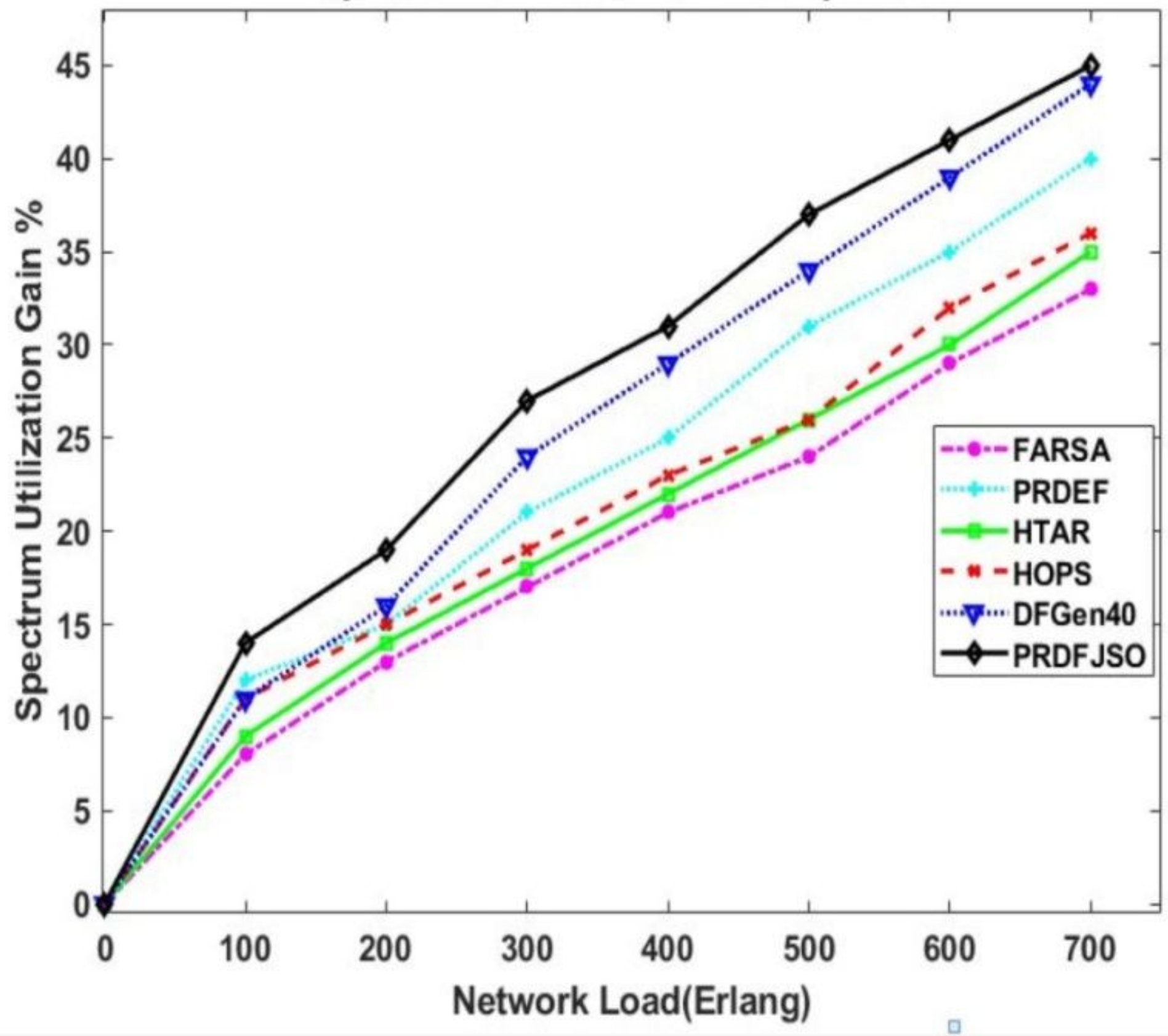

Figure 6

The Spectrum Utilization Gain Comparison in NSFNET. 
Spectrum Utilization Gain comparison in USNET

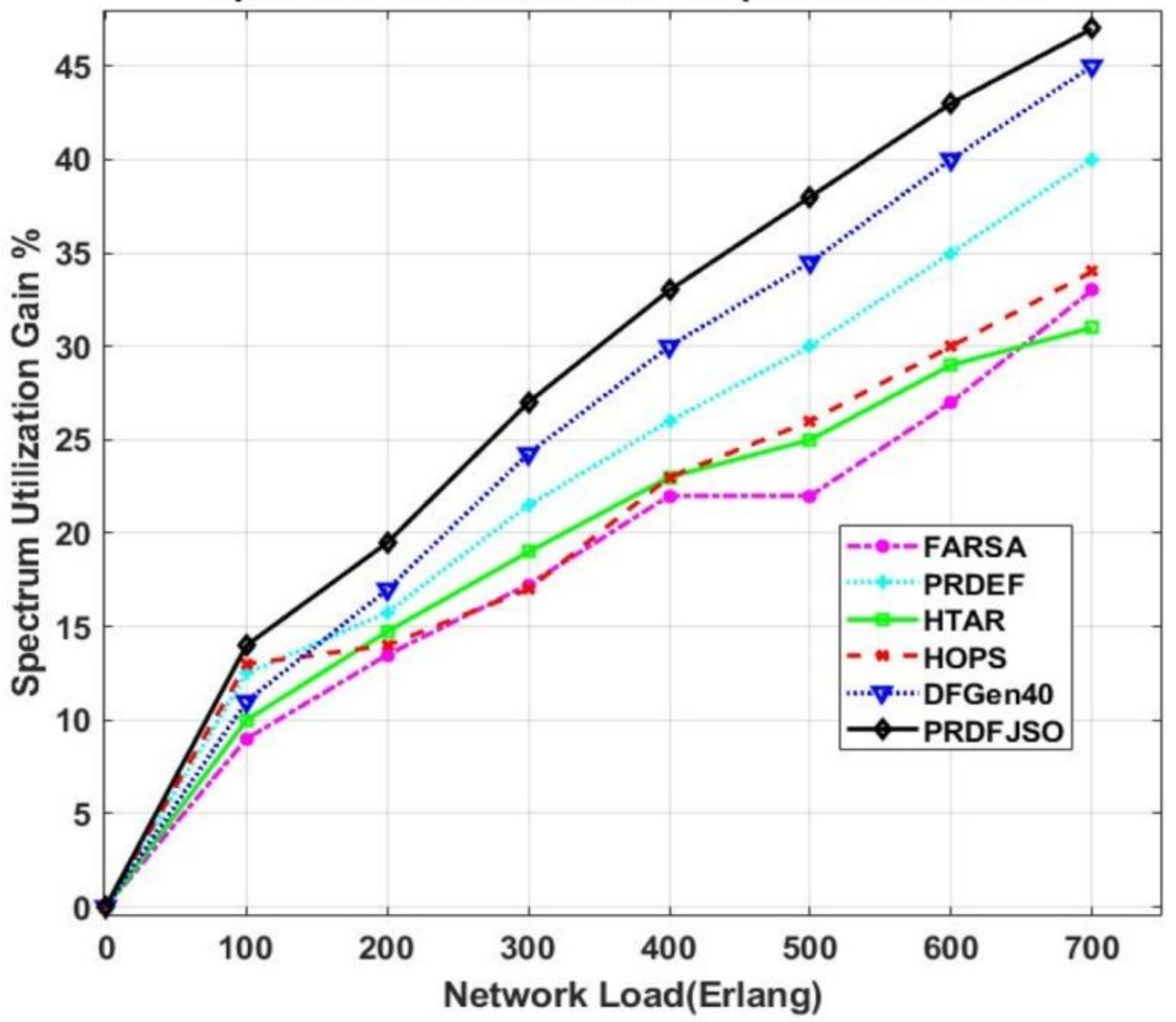

Figure 7

The Spectrum Utilization Gain Comparison in USNET. 


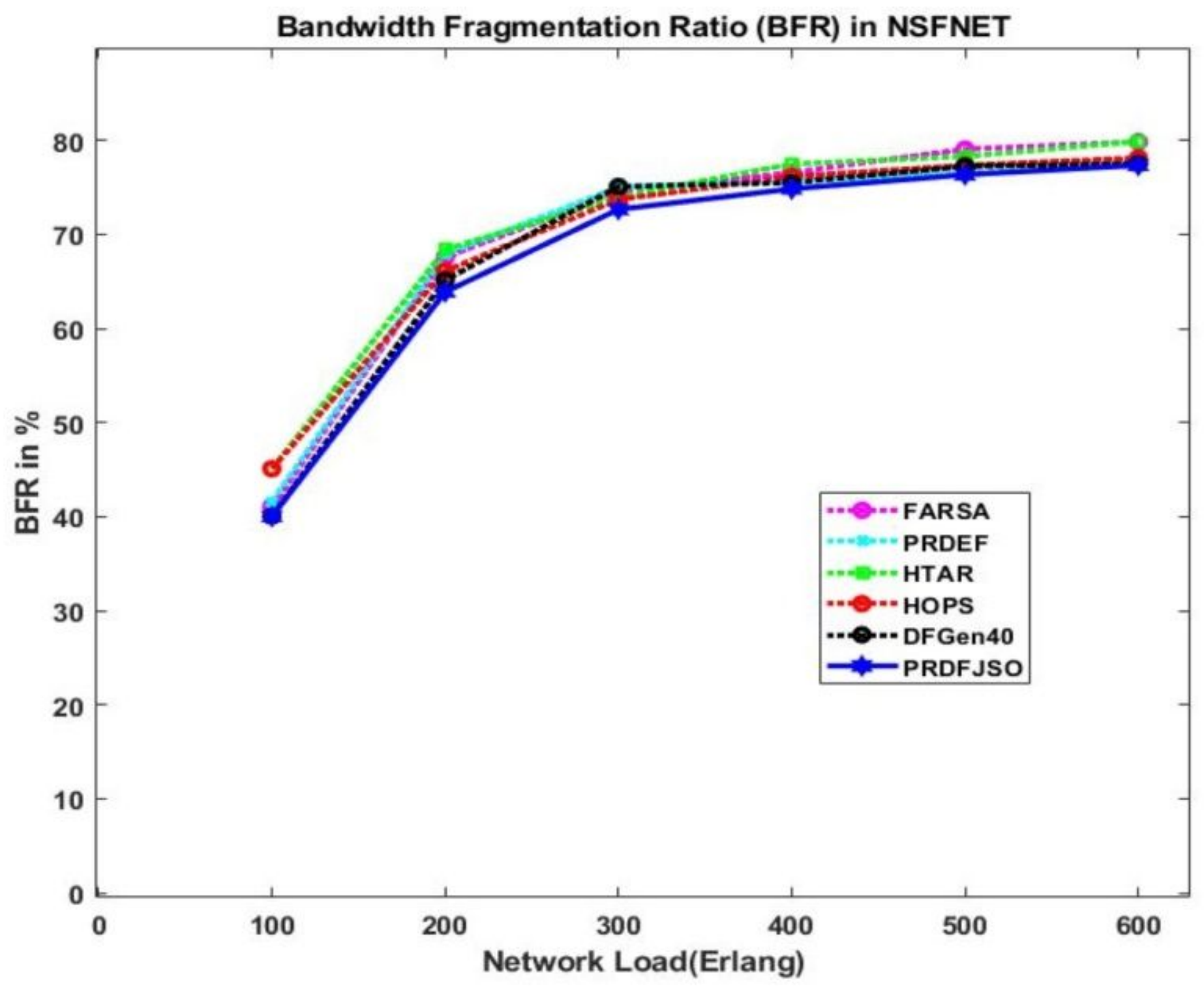

Figure 8

Bandwidth Fragmentation Ratio (BFR) Comparison in NSFNET 


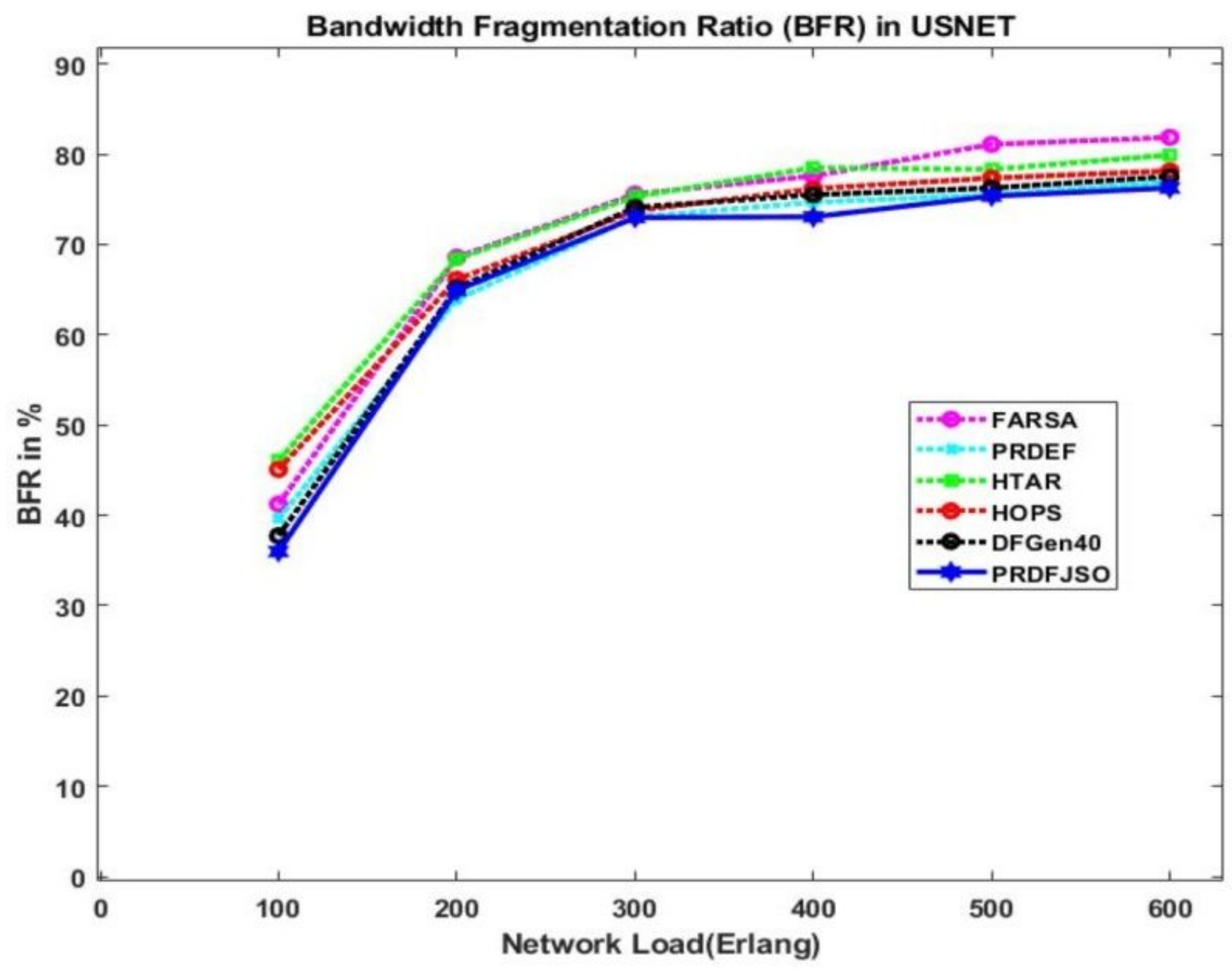

Figure 9

Bandwidth Fragmentation Ratio (BFR) Comparison in USNET 


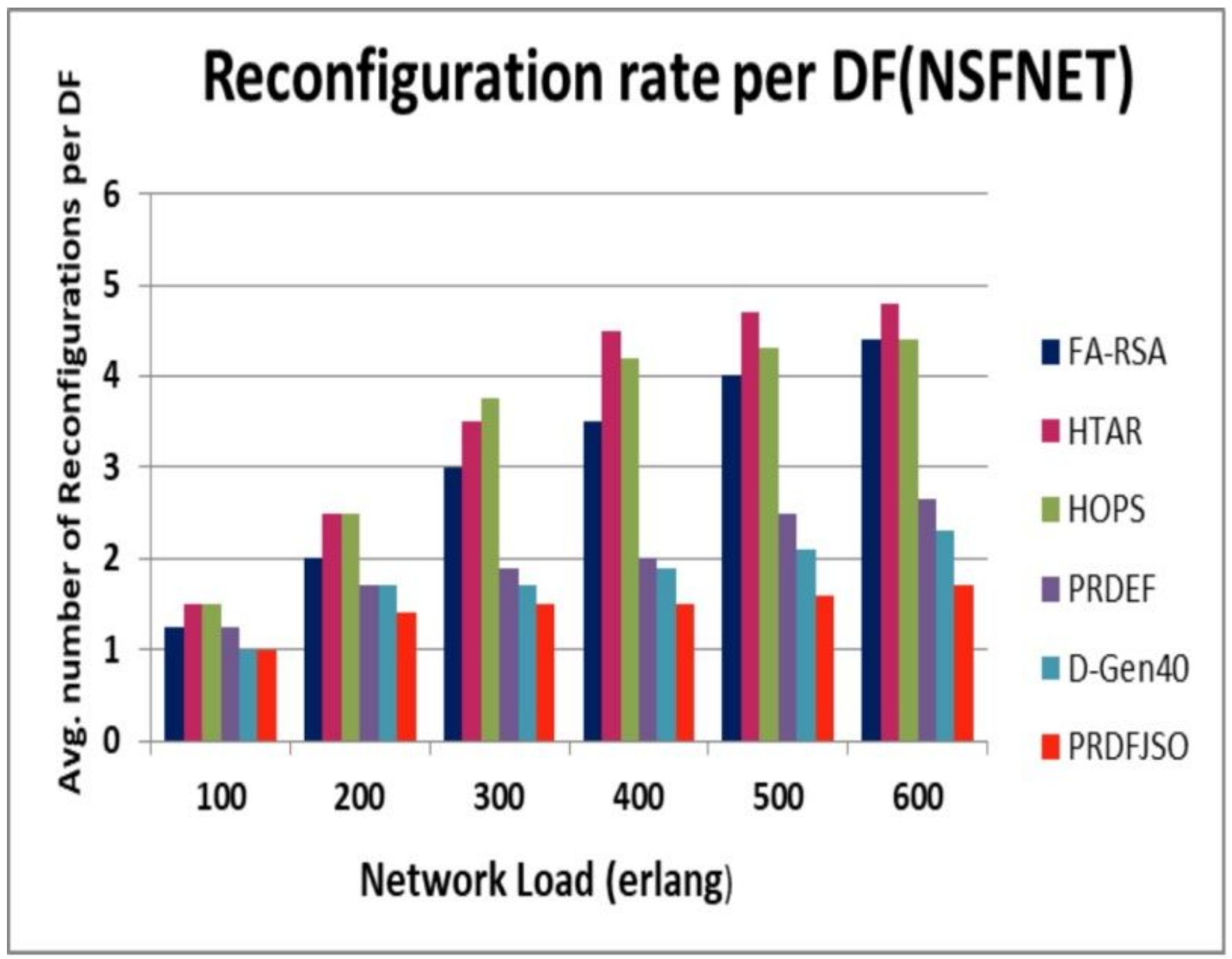

Figure 10

Average number of reconfigured connections for every performed DF (NSFNET). 